\title{
The Integration of Positron Emission Tomography With Magnetic Resonance Imaging
}

\section{First-generation systems that combine these two techniques have been developed offering new opportunities for integrated structural-functional imaging in vivo.}

\author{
By Simon R. Cherry, Fellow IeEe, Angelique Y. Louie, and Russell E. Jacobs
}

\begin{abstract}
A number of laboratories and companies are currently exploring the development of integrated imaging systems for magnetic resonance imaging (MRI) and positron emission tomography (PET). Scanners for both preclinical and human research applications are being pursued. In contrast to the widely distributed and now quite mature PET/computed tomography technology, most PET/MRI designs allow for simultaneous rather than sequential acquisition of PET and MRI data. While this offers the possibility of novel imaging strategies, it also creates considerable challenges for acquiring artifact-free images from both modalities. This paper discusses the motivation for developing combined PET/MRI technology, outlines the obstacles in realizing such an integrated instrument, and presents recent progress in the development of both the instrumentation and of novel imaging agents for combined PET/MRI studies. The performance of the first-generation PET/MRI systems is described. Finally, a range of possible biomedical applications for PET/MRI are outlined.
\end{abstract}

KEYWORDS | Biomedical imaging; magnetic resonance imaging; multimodality imaging; positron emission tomography

\section{INTRODUCTION}

Magnetic resonance imaging (MRI) and positron emission tomography (PET) have established themselves as very powerful and flexible imaging technologies. MRI provides exquisite anatomic images, with high spatial resolution (as

Manuscript received June 1, 2007. This work was supported in part by the National Institutes of Health under Grants R01 EB000993 and U24 RR021760 and the Gordon and Betty Moore Foundation.

S. R. Cherry and A. Y. Louie are with the Department of Biomedical Engineering, University of California-Davis, Davis, CA 95616 USA (e-mail: srcherry@ucdavis.edu; aylouie@ucdavis.edu).

R. E. Jacobs is with the Beckman Institute, California Institute of Technology, Pasadena, CA 92715 USA (e-mail: rjacobs@caltech.edu).

Digital Object Identifier: 10.1109/JPROC.2007.913502 good as tens of micrometers for preclinical studies and $\sim 1 \mathrm{~mm}$ for clinical studies) and excellent soft tissue contrast. Image contrast can often be selectively enhanced utilizing a range of passive contrast agents, some based on paramagnetic atoms, particularly gadolinium [1], some on superparamagnetic iron oxide particles [2], and others on hyperpolarized gases such as xenon [3]. MRI can interrogate aspects of physiology; for example, functional MRI (fMRI) takes advantage of the physiologic consequences of neuronal activation (the "BOLD" effect) to visualize task-dependent responses [4]. MRI is also capable of reporting on abundant molecular targets and pathways through the use of a range of targeted imaging probes and contrast mechanisms [5]. Finally, measurement of the chemical shifts in the resonance signal of protons, as well as nuclides such as ${ }^{31} \mathrm{P}$ and ${ }^{19} \mathrm{~F}$, allows the abundance of many interesting metabolic products and mass levels of drugs to be detected. With magnetic resonance spectroscopic imaging (MRSI), these compounds can be mapped spatially [6], albeit at a relatively coarse scale compared with the resolution of conventional anatomic MRI.

PET has tremendous flexibility in interrogating biologic processes using a range of targeted probes that include radio-labeled small molecules (e.g., receptor ligands, enzyme substrates), peptides, antibodies, and cells [7], [8]. With extremely high sensitivity, PET allows the quantitative imaging of even relatively low abundance targets without introducing mass effects. PET radionuclides range from short-lived biologically relevant isotopes such as ${ }^{15} \mathrm{O}\left(\mathrm{T}_{1 / 2}=2 \mathrm{~min}\right)$ and ${ }^{11} \mathrm{C}\left(\mathrm{T}_{1 / 2}=20 \mathrm{~min}\right)$ to the clinically ubiquitous ${ }^{18} \mathrm{~F}\left(\mathrm{~T}_{1 / 2}=110 \mathrm{~min}\right)$ and longer lived radiohalogens and radiometals such as ${ }^{64} \mathrm{Cu}\left(\mathrm{T}_{1 / 2}=12.6 \mathrm{~h}\right)$, ${ }^{76} \mathrm{Br}\left(\mathrm{T}_{1 / 2}=16.1 \mathrm{~h}\right)$, and ${ }^{124} \mathrm{I}\left(\mathrm{T}_{1 / 2}=4.2 \mathrm{~d}\right)$. While the sensitivity for detection is several orders of magnitude better than MRI, the spatial resolution is significantly inferior, ranging from around $1 \mathrm{~mm}$ for preclinical PET scanners to 4-6 $\mathrm{mm}$ for human whole-body PET scanners. 
PET and MRI both utilize radiation that penetrates tissue well; thus these techniques have translational applications that bridge from basic biological research and preclinical studies in animal models to clinical research, clinical trials, and diagnostic use in humans. As PET and MRI occupy quite different positions in resolution/ sensitivity space, neither PET nor MRI alone is sufficient to tackle the broad range of questions that might be of interest in a research or diagnostic study. Therefore they are often used in combination. Indeed, it is quite common for both PET and MRI studies of a subject to be acquired on separate scanners and then spatially coregistered through advanced image alignment algorithms [9]. This has been particularly effective in the brain, where tissue remains relatively fixed in position because of the skull allowing simple rigid body transformations to be used to align the datasets with high precision. Outside the brain, however, software registration of the two datasets is far from trivial and typically requires elastic transformations to account for differences in patient pose and changes in internal geometry due to dynamic processes within the body (e.g., movement of stomach contents, filling of the urinary bladder). Depending on the information content of the two datasets, it may not be possible to accurately align such studies. This raises the question as to whether an integrated PET/MRI instrument that can simultaneously, or near simultaneously, acquire the two datasets in a fixed geometry might be a better solution. In addition to the obvious advantage of facilitating precise anatomic localization of signals detected in PET studies (the primary role for PET/ computed tomography (CT) scanners), such an instrument would open up novel opportunities, for example, the temporal correlation of PET and MRSI studies, the imaging of two molecular targets simultaneously using distinct PET and MRI imaging probes, or simultaneous high-sensitivity (PET) and high-resolution (MRI) imaging of a target using a single imaging probe that is both PET and MR visible.

In this paper, we discuss the challenges and obstacles in developing PET/MRI instruments and review the technologies being developed for integrated PET and MRI scanners, focusing on an MRI-compatible PET insert developed at the University of California, Davis, in collaboration with the California Institute of Technology. We then discuss the development of novel dual-modality targeted contrast agents that could be used with such a system. Finally, we speculate on some of the applications for such a device, in both the clinical and the preclinical arenas, and outline remaining opportunities and challenges that still need to be addressed.

\section{INTEGRATED PET/MRI SYSTEMS}

\section{A. General Considerations}

Some of the essential features of an MRI scanner are: i) a high and extremely homogeneous (typically a few ppm) main magnetic field usually in the range of 1.5-11.7 T; ii) smaller magnetic field gradients (typical ranges of 20 to $100 \mathrm{mT} / \mathrm{m}$ ) that are applied across the imaging field of view and that are rapidly switched on and off; and iii) relatively high power radio-frequency pulses that are used to excite the sample at the resonance frequency of the nuclei of interest. For protons, this ranges from $64 \mathrm{MHz}$ at $1.5 \mathrm{~T}$ to $500 \mathrm{MHz}$ at $11.7 \mathrm{~T}$.

PET scanners typically consist of annular arrays of scintillation detectors, where the $511 \mathrm{keV}$ annihilation photons resulting from $\beta^{+}$(positron) decay are converted into visible light by interaction in dense, fast scintillator materials, and this scintillation light is then converted to an electrical signal, commonly using a photomultiplier tube (PMT). By way of example, the scintillator lutetium oxyorthosilicate (LSO) emits on the order of 12000 photons centered around $420 \mathrm{~nm}$ when $511 \mathrm{keV}$ of energy is deposited. Depending on the geometry and surface treatment of the scintillator and the application of external reflectors, something on the order of $20 \%-60 \%$ of the scintillation light photons reach the photocathode of the PMT, where, with a quantum efficiency of roughly $20 \%$ at $420 \mathrm{~nm}$, several hundred photoelectrons are typically produced. This signal is amplified by a factor of roughly $10^{6}$ by the PMT, producing a transient current in the milliamp range at the PMT anode. A PET scanner may consist of many thousands of scintillator detector elements, read out by tens or hundreds of PMTs.

In integrating these two imaging technologies, several possible impediments immediately come to mind. A PET scanner within or close to the magnet could interfere with the ability to acquire MR data by disturbing the homogeneity of the main magnetic field or the linearity of the gradient fields beyond the level that can be accurately corrected by shimming. If components of the PET scanner are placed within the magnet, then susceptibility artifacts caused by the materials comprising these components, or the possibility of eddy currents being generated in these components, become a concern. Another critical area to consider is RF radiation emitted by the PET scanner electronics, particularly if there is any significant power radiated at the resonance frequencies of interest that would interfere with the MR measurement. There also are a number of mechanisms by which the MRI scanner might interfere with PET data acquisition. This includes the effect of the main magnetic field and gradient fields on the photodetector that is used to read out the scintillator. It is worth noting that PMTs are particularly susceptible to magnetic fields, with most PMTs showing significant gain changes in fields as modest as a few milliTesla. The generation of eddy currents in critical components of the PET pulse processing pathway, and RF interference caused by rapid changing of gradient fields or pulsing of RF excitation, could also destroy the low-amplitude PET signals. These considerations demonstrate that a functional integration of these two modalities, with performance 


\section{(a) "tandem" PET/MRI}

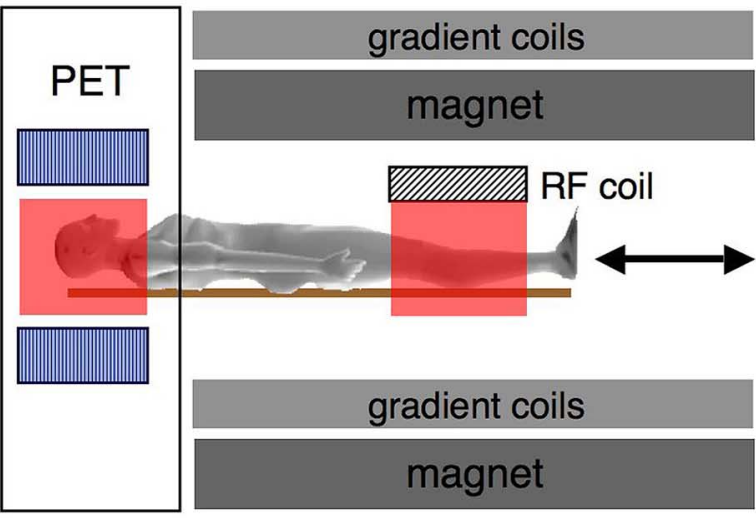

(b) "integrated" PET/MRI

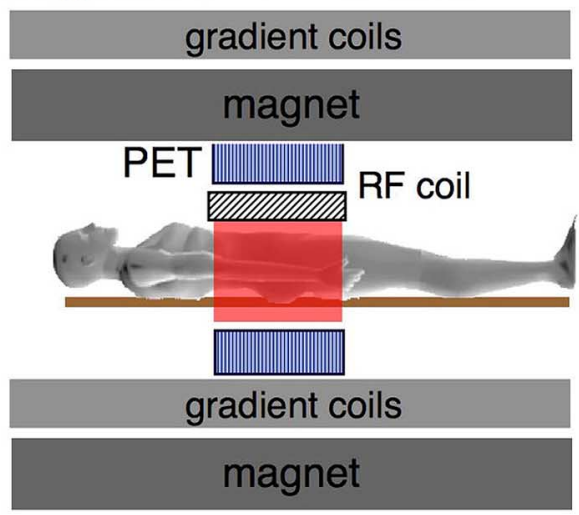

Fig. 1. Two possible geometries for combined PET/MRI scanners. (a) The "tandem" configuration is similar to most PET/CT scanners in that the two scanners are axially displaced and PET and MRI studies would be acquired sequentially. (b) The "integrated" configuration involves placing the PET scanner within the magnet such that the same region can be imaged by PET and MRI simultaneously.

consistent with standalone PET or MRI devices, is challenging. Nonetheless, over the past decade, there has been considerable progress as will be described below.

There are two main geometries one can consider in combining PET and MRI into a single imaging system (Fig. 1). In the tandem configuration, a PET scanner may be placed just outside the magnet, and PET/MRI imaging would be accomplished by moving the patient sequentially through the two imaging devices. This is typically the geometry used in clinical PET/CT scanners. The advantage of this configuration is that by separating the two systems axially, possible interference can be minimized, the PET scanner design is not subject to geometric constraints imposed by the bore size of the MR system, and existing PET and MRI systems might be able to be used with relatively little modification. It is also likely the cheapest way to realize PET/MRI systems and the approach least likely to lead to compromises in image quality with either modality. For all these possible advantages, it is interesting to note that none of the PET/MRI scanners actually built to date employs this approach. The second configuration involves building a PET scanner within the bore of the MR magnet. The advantages of this integrated approach are that the PET and MRI systems view the same region of the subject and, if interference is eliminated, simultaneous PET/MRI imaging is possible. This is attractive from a number of perspectives. In considering clinical applications, it dramatically improves throughput (both PET and MRI are relatively slow imaging techniques compared with CT) and, for research applications, it opens up enticing prospects of temporally correlating dynamic PET studies with contrast (with passive or targeted agents) MR studies or MR spectroscopy. However, the technical challenges are now significant. The PET scanner must be reengineered to fit within the bore of the magnet, interference between PET and MR systems that are colocated must be negligible, and compromises in imaging performance of one or both modalities become a major concern.

\section{B. Early Concepts: Effects of High Magnetic Fields on Positron Range}

The concept of performing PET studies within a highfield magnet dates back at least to the early 1990s, when Hammer and colleagues [10], [11] studied the effect that a high static magnetic field would have in reducing the range of positrons prior to their annihilation. PET images actually reflect the spatial distribution of the positron annihilation sites within the body, and this differs from the site of radioactive decay (and therefore the location of the radio-labeled molecule) by the distance the positron travels prior to annihilation. The positron range depends on the energy distribution of the emitted positrons, and this varies widely between positron-emitting radionuclides. When imaging high-energy positron-emitting radionuclides on very high-resolution PET scanners, positron range can be a limiting factor in the achievable spatial resolution. Placing the sample in a strong magnetic field constrains the positron travel in the plane perpendicular to the magnetic field lines and, therefore, reduces positron range in two of three dimensions. MRI scanners are a natural and available source for such a strong magnetic field, and the concept of PET/MRI was therefore explored. A number of experimental studies and Monte Carlo simulations have studied this effect [11]-[13], and for fields above $4.7 \mathrm{~T}$ and radionuclides such as ${ }^{15} \mathrm{O},{ }^{68} \mathrm{Ga}$, and ${ }^{82} \mathrm{Rb}$, the effects can be significant (Fig. 2). However, for the most commonly utilized PET radionuclides ${ }^{18} \mathrm{~F}$ and ${ }^{11} \mathrm{C}$, the effects are small because they emit relatively lowenergy positrons. The effects of positron range are likely only seen in the very highest resolution preclinical PET scanners, and even here, they are generally still not the dominant source of resolution loss [14]. 


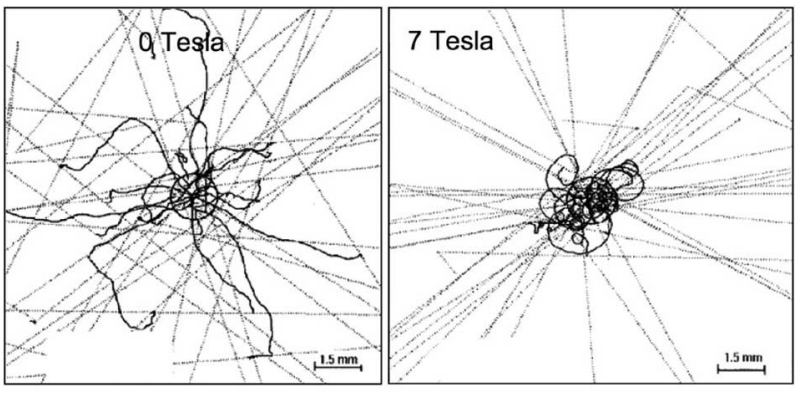

Positron Energy $3 \mathrm{MeV}$

Fig. 2. Monte Carlo simulation showing the effect of a $7 \mathrm{~T}$ magnetic field on the trajectories and annihilation locations of $3 \mathrm{MeV}$ positrons. Reproduced with permission from [13].

C. First Attempts at Simultaneous PET/MRI Imaging

The magnetic field sensitivity of the PMTs used in most PET detectors was well known, and therefore the earliest attempts at detecting PET radionuclides within a magnet focused on using light guides placed in between the scintillator and the PMT. The purpose of the light guide was to pipe scintillation light from scintillator crystals placed within the MRI scanner to shielded PMTs, placed outside the magnet in a location where the fringe field had dropped to levels low enough for operation [15], [16]. A system that allowed the detection of total radiotracer activity within an object placed inside of a nuclear magnetic resonance (NMR) spectrometer was developed, allowing dynamic radio-assay studies to be performed concurrently with NMR spectroscopic measurements [16]. However, this system provided no spatial information regarding the distribution of the radiotracer within the object.

By replacing the light guides with flexible optical fibers, and paying careful attention to maximizing scintillation light collection and transmission along the fiber, it became possible to consider placing larger numbers of scintillation detectors within the bore of the magnet, providing sufficient sampling for images to be produced. The first MR-compatible PET system had a single ring of just 48 LSO scintillators, and with a bore size of $3.8 \mathrm{~cm}$ could only be used for imaging small test objects. Nonetheless, it did demonstrate for the first time that PET and MR images could be acquired simultaneously with little apparent interference between the two systems [17]. A subsequent version [18] increased the number of scintillator elements to 72 and had a bore size appropriate for small animal and isolated organ imaging (Fig. 3). This system was extensively used for temporally correlated PET and NMR measurements of isolated perfused rat hearts at $9.4 \mathrm{~T}$ [19], and a series of phantom studies demonstrated the lack of any significant interference when conducting simultaneous PET and MRI studies at several different field strengths [20].

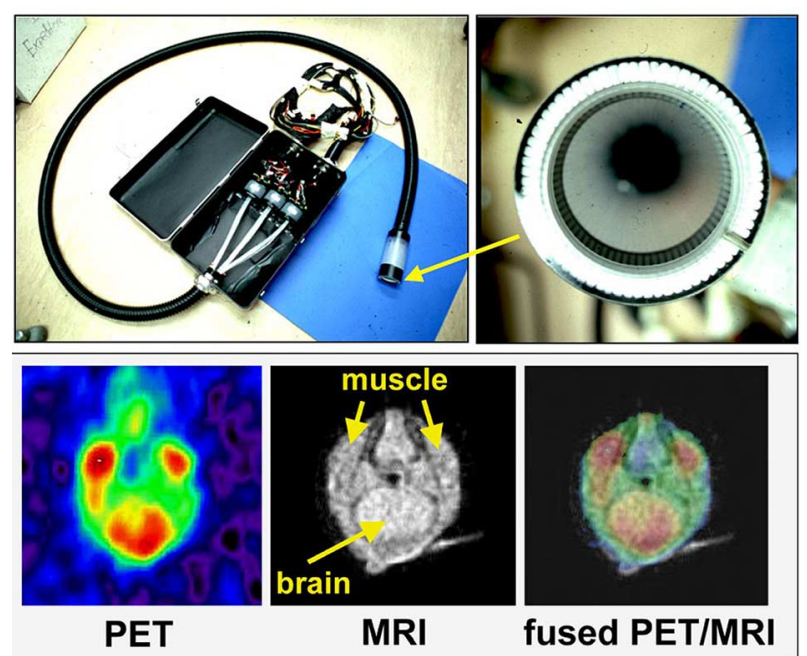

Fig. 3. (a) Early MRI-compatible PET insert featuring (b) a ring of 72 LSO scintillator elements coupled via 3-m-long optical fibers to multichannel PMTs. (c) First simultaneous in vivo PET and MRI images acquired with this system. Adapted with permission from [139].

\section{PMT-Based PET Systems Inside Existing MRI Scanners}

While these very first systems were sufficient for proofof-concept studies, they had significant limitations in terms of their PET performance for routine in vivo use. In particular, the sensitivity of the devices (the fraction of radioactive decays leading to a valid coincidence event) was very low because of the small number of detectors. Furthermore, the long optical fibers attenuated much of the scintillation light, reducing the energy and timing resolution of the PET measurement. Recently, a higher density system has been constructed along similar lines with 416 detectors, improving resolution uniformity and sensitivity of the PET insert relative to earlier attempts [21]. This PET insert, however, is still limited to producing a single transverse slice through the object. PMT-based approaches using long optical fibers have also been used to obtain limited angle tomographic PET images simultaneously with MRI in vivo [22].

\section{E. Novel MRI Scanner Designs for PET/MRI}

The methods discussed so far have involved designing a PET insert to fit within existing MRI systems. An alternative approach is to change the design of the MR system so that it can better accommodate the detectors comprising a PET system. Two different ideas that involve modification of the MRI magnet are currently being pursued.

The first utilizes a split magnet design [23], where the PET detectors will reside in the gap between the magnets (Fig. 4). The PET detectors still consist of scintillator elements coupled through long optical fibers to PMTs; however, in this configuration, the fiber optics emanate radially from the magnet, allowing many more PET 

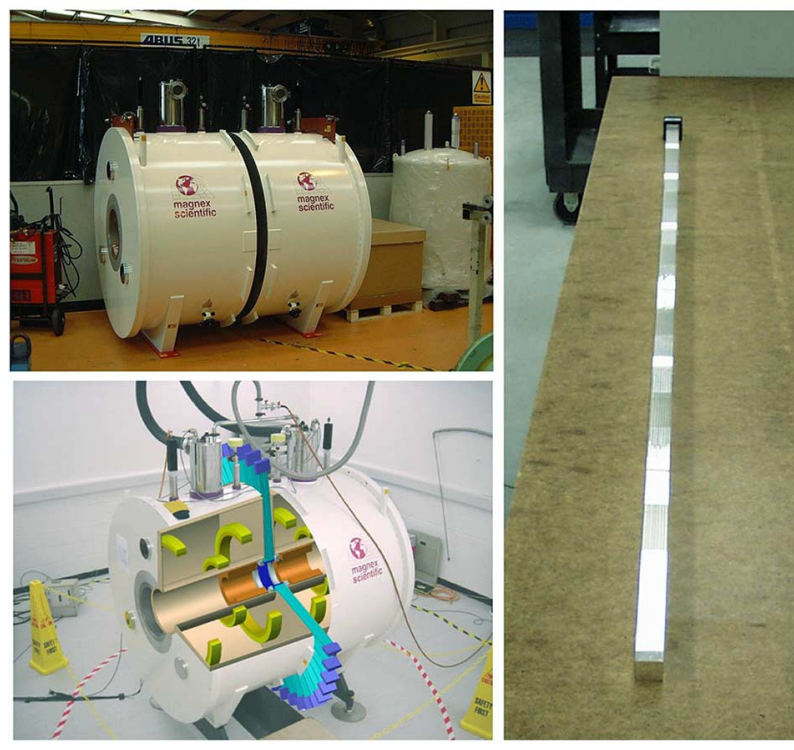

Fig. 4. (a) Split magnet design for combined PET/MRI. (b) The PET detectors will be located in the gap with the optical fibers emanating radially as shown. (c) View of (front) a single PET detector module with scintillator array connected to (back) a position-sensitive PMT by optical fibers. Adapted with permission from [23].

detectors to be packed into the system [Fig. 4(b)]. This will allow the sensitivity of the PET component to rival that of standalone preclinical PET systems and provides imaging of a full three-dimensional volume rather than an individual slice. However, the loss of scintillation light through the long optical fibers still compromises the energy and timing resolution of the PET component (not critical for preclinical imaging, but this could be a major drawback for scaling up to humans), and the field strength of the MR system is only $1.0 \mathrm{~T}$, limiting perhaps some of the more advanced MR applications one might be interested in pursuing in combination with PET.

The second approach involves a novel field-cycled magnet [24], [25] in which the polarizing and readout magnetic fields can be rapidly cycled on and off, allowing PET data acquisition to be interleaved with MR data acquisition and to occur at times when the magnetic field is zero. This permits PMTs to be used without long optical fiber connections, avoiding the light losses described in earlier designs. A further advantage is that the polarizing field does not need to be homogeneous. However, the effective sensitivity of the PET scanner is lower than the absolute sensitivity because data can only be collected for a certain fraction of the total acquisition time. Furthermore, the field strength of the MR system is low, once again limiting the range of possible MR applications.

\section{F. Avalanche Photodiodes for PET/MRI}

The problems created by the magnetic field sensitivity of PMTs can be avoided by replacing them with avalanche photodiodes (APDs). These silicon devices convert light photons into electron-hole pairs that are then amplified by a gain factor on the order of 100-1000. Because the charge generation and multiplication take places in a very thin layer of silicon, with a very high electric field across it, electron trajectories are virtually unaffected by even very high magnetic fields [26]. This makes them attractive for PET/MRI systems. With improvements in their stability and more widespread availability, several laboratories and companies have been exploring their use. Challenges in using APDs include the strong dependence of their gain on temperature and bias voltage and their lower overall gain compared with PMTs, which reduces signal amplitude and generally requires preamplifiers to be placed immediately adjacent to the devices.

In the PET insert recently developed at the University of California, Davis, position-sensitive APDs (PSAPDs) are used to read out LSO scintillator arrays using short optical fiber bundles such that the PSAPDs and their readout electronics reside within the bore of the magnet but outside of the imaging field of view of the MRI (Fig. 5). This $60 \mathrm{~mm}$ outer diameter insert has a total of $1024 \mathrm{LSO}$ elements with dimensions of $1.43 \times 1.43 \times 6 \mathrm{~mm}^{3}$ that are arranged in eight rings and provides a field of view (FOV) of $35 \mathrm{~cm}$ in the transverse direction and $12 \mathrm{~mm}$ in the axial direction [27]. The spatial resolution of the reconstructed PET images is $1.2 \mathrm{~mm}$, and the sensitivity at the center of the FOV is $\sim 0.3 \%$. Phantom experiments inside a Bruker $7.0 \mathrm{~T} / 30$ BIOSpec animal MRI system demonstrate no significant effect of the PET insert on the performance of the MRI system. Conversely, with the exception of a very small drop in sensitivity $(<10 \%)$ that is observed when running some pulse sequences, the MR system has no observable effect of the PET system. This small loss in sensitivity is likely due to a loss of PET data during times when the gradients are rapidly switching. More demanding MR pulse sequences, including echo planar imaging (EPI), high-resolution MRI, and localized spectroscopy have also been successfully conducted in the presence of the PET insert. This PET system has now been used for a range of in vivo imaging studies where MRI and PET data are acquired simultaneously.

A similar approach has been taken by researchers at the University of Tübingen, except in this case, the optical fiber connection has been completely eliminated and the APDs and associated electronics are coupled directly behind the scintillator arrays within the MRI field of view [28]. Initial results from this system, including dynamic PET and MRI measurements, also have clearly demonstrated the feasibility of simultaneous PET and MR imaging. APD approaches for preclinical PET/MRI are also being investigated by others [29]. It remains to be seen whether there is any advantage from the MRI perspective of keeping the APD readout outside of the imaging field of view or whether the fiber-optic coupling can be abandoned for even the most demanding MRI measurements, which 

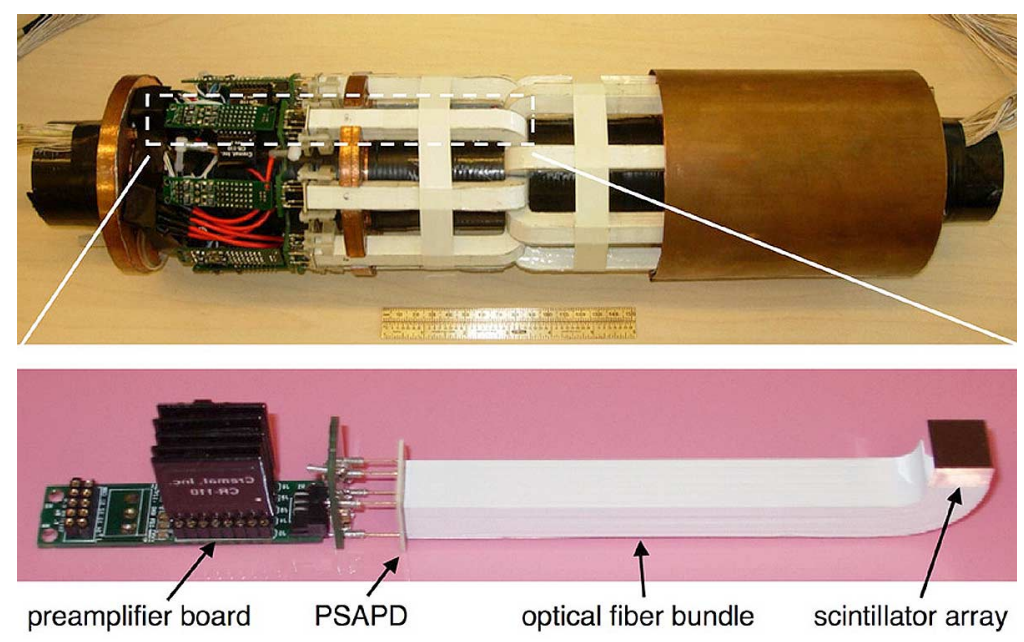

Fig. 5. Photograph of (top) PET insert for preclinical MRI scanner based on position-sensitive APD readout of $1.5 \times 1.5 \times 6$ mm $^{3}$ LSO scintillator elements via short bundles of optical fibers. Lower photograph shows individual detector module. Adapted with permission from [27].

would give the best possible performance from the PET perspective.

All the systems discussed so far have been designed for preclinical imaging, partly because there are significant motivations for simultaneous PET/MRI studies in the research setting and partly because the smaller volume of detectors needed in the magnet for rodent imaging makes this an attractive starting point. Recently, the first human PET/MRI scanner has been developed by Siemens [30], also using the APD approach, in a very similar detector configuration to that in the preclinical system at the University of Tübingen. Impressively, the $35.5-\mathrm{cm}-$ diameter insert consists of 23040 LSO scintillator elements read out by 1440 APDs with an axial FOV of $19 \mathrm{~cm}$ (Fig. 6). This PET insert is installed in the Siemens Trio $3 \mathrm{~T}$ scanner, and simultaneous in vivo PET and MR imaging of the human brain has been demonstrated, with a spatial resolution in the PET images of about $2.5 \mathrm{~mm}$.

\section{MULTIMODAL CONTRAST AGENTS}

The use of multiple modalities for a common imaging problem does not always require the use of multiple contrast agents detected by each modality. Many reported studies use combinations of PET with soft tissue contrast methods such as CT and MRI, where the CT or MRI may be conducted with or without contrasts agents. In this section, we provide an overview of the application of contrast agents in multimodality imaging studies. We first briefly review PET imaging with MRI using combinations of contrast agents and then highlight development of "multimodal" agents ranging from $\mathrm{MRI} /$ optical to PET/MRI.

\section{A. Combined Modality Imaging With Contrast Agents}

The earliest research using multiple modalities was performed primarily for the sake of validating one modality

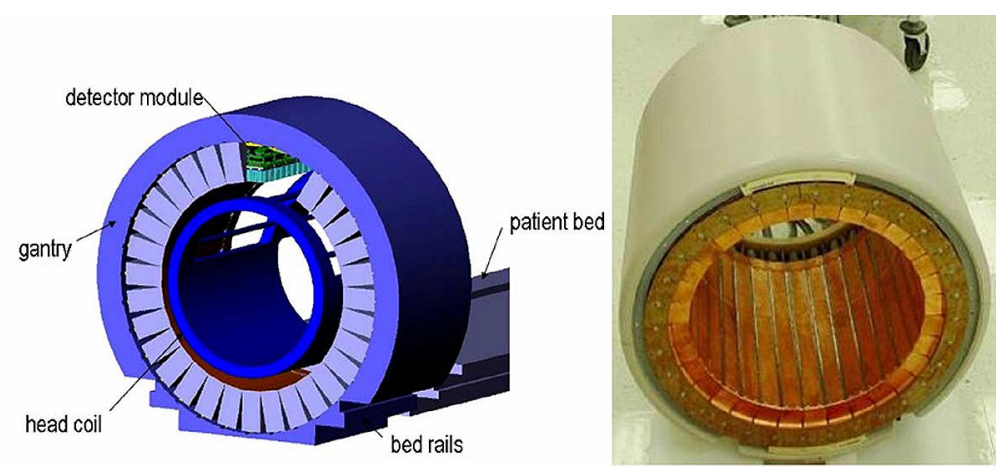

Fig. 6. Schematic and photograph of a PET insert developed for human brain imaging. Detectors are based on LSO scintillator read out by arrays of APDs. (Courtesy of I. Panagiotelis, Siemens Medical Solutions.) 
through comparison with the other, rather than with the intent of combining information. Nonetheless, these comparisons of multiple imaging modalities may have sparked interest in using the imaging methods more synergistically. Comparisons of PET performance with MRI for imaging of brain tumors has been one of the most extensively reported areas of study. MRI is routinely employed for morphological imaging of brain tumors, typically with the use of a gadolinium-based agent for contrast enhancement. Leakage of such a blood pool contrast agent through the compromised neovasculature in the tumor creates areas of enhanced contrast that demarcate the tumor vascular boundaries. However, the condition and aggressiveness of the tumor cells is not revealed by this method. Metabolic information would be more diagnostic of tumor stage, but the use of ${ }^{18} \mathrm{~F}$-fluorodeoxyglucose (FDG) PET for imaging brain tumors is not ideal, as normal brain tissue also has very high levels of glucose metabolism; nevertheless, many studies have demonstrated the utility of using FDG-PET to stage tumors or assess the success of therapy [31]-[34]. Some of these comparative studies have discovered that the different modalities yield complementary information, thereby setting the stage for future work to further explore this synergistic effect. For example, a recent study comparing ${ }^{18} \mathrm{~F}$-fluorothymidine (FLT) and ${ }^{11} \mathrm{C}$-methione (MET) PET with gadolinium-enhanced MRI in patients with glioma discussed the use of gadolinium-enhanced MRI to determine the integrity of the blood-brain barrier, MET to identify tumor invasion to neighboring tissue, and FLT, with its longer half-life (relative to MET) for more accurate assessment of cell proliferation [35]. Work in other disease systems such as breast tumors [36], lymph node metastasis [37], and inflammation [38], [39] has observed greater accuracy for diagnoses based on the results of more than one imaging method.

The brain applications described above all use clinically approved gadolinium-enhanced contrast methods for morphological characterization. In addition to the $\mathrm{T}_{1}$ and $\mathrm{T}_{2}$ MR imaging methods used above, more quantitative MR imaging methods can be employed to assess values such as regional cerebral blood volumes (rCBVs). Brain gliomas have been imaged with combinations of MET and dynamic susceptibility contrast perfusion MRI to measure rCBV [40]. In this application, both MRI and PET were used to determine the proliferative and malignant potential of gliomas and the results from each found to closely correlate. MRI predicts tumor grade based on the rCBV, which is higher in high-grade gliomas, while MET-PET marks proliferating cells.

In addition to the use of MRI for simple anatomical reference, the power and versatility of MRI to capture dynamic information have also been exploited for complementary imaging in conjunction with PET. Through the use of appropriate imaging parameters, MRI can be tuned to assess the dynamic movement of nuclei, i.e., diffusion, in an image, thus providing time-dependent functional information. This aspect of MRI has been widely used in cardiovascular applications in conjunction with PET [41], [42]. For example, PET and gadolinium-enhanced MRI have been applied to assess defective glucose metabolism in infarcted tissue and cardiac function, respectively, in both animal models and human patients [43], [44]. The results of the studies in rat models illustrate that MRI measurements of scarring can complement FDG-PET determination of myocardial viability to reveal the stage of ischemic damage to the heart [43]. In the human patients, ${ }^{15} \mathrm{O}$-water PET was used to assess perfusion, while delayed contrast enhancement cardiac MRI was used to identify fibrotic tissue in patients with chronic myocardial infarction [43]. The greater the amount of fibrosis and lower the perfusion of tissue by water, the more severe the disease stage. Both MRI and PET have been used to assess myocardial viability [45].

The examples above have primarily treated the use of multiple modalities as separate imaging methods and compared images obtained by the various methods without any attempt to overlay or spatially register the images. With increasing interest in truly combining imaging methods, there has been an increase in reports of coregistered and simultaneously acquired, coregistered imaging studies.

\section{B. Fused PET and MRI Images Using Contrast Agents}

Comparison of images between data sets obtained by different imaging methods is considerably easier than coregistering the images, but correlation of information for diagnosis can require the greater accuracy of coregistered methods. Methods to coregister data are a topic of intense investigation and constant improvement [46], [47]. Applications in the brain, for example, use coregistration of images to precisely characterize drug uptake and transport [48].

While applications in the brain for combined radionuclide-based and MRI have been explored for years, a newer application generating some excitement has been cell tracking. Recent studies have used coregistered images from ${ }^{111}$ In-SPECT [49] and MRI, or ${ }^{124}$ I-PET and MRI [50], to track cell homing in cardiovascular and cancer applications, respectively. The SPECT/MRI studies use MRI to provide anatomical mapping against ${ }^{111} \mathrm{In}$-oxyquinoline labeled stem cells grafted to infarcted rat myocardium while simultaneously acquiring $99 \mathrm{~m}_{\mathrm{Tc}}$-sestamibi maps of perfusion deficient regions. The two radionuclide signals were simultaneously acquired by using separate energy windows. Fig. 7 shows the three coregistered images, with stem cells and perfusion deficient regions mapping to the akinetic region identified by MRI. In the discussion, the authors comment on errors in coregistration that occurred as a result of acquiring the MRI and SPECT images several days apart, during which time the myocardium was undergoing remodeling. Simultaneous imaging with MRI and PET or SPECT would have 


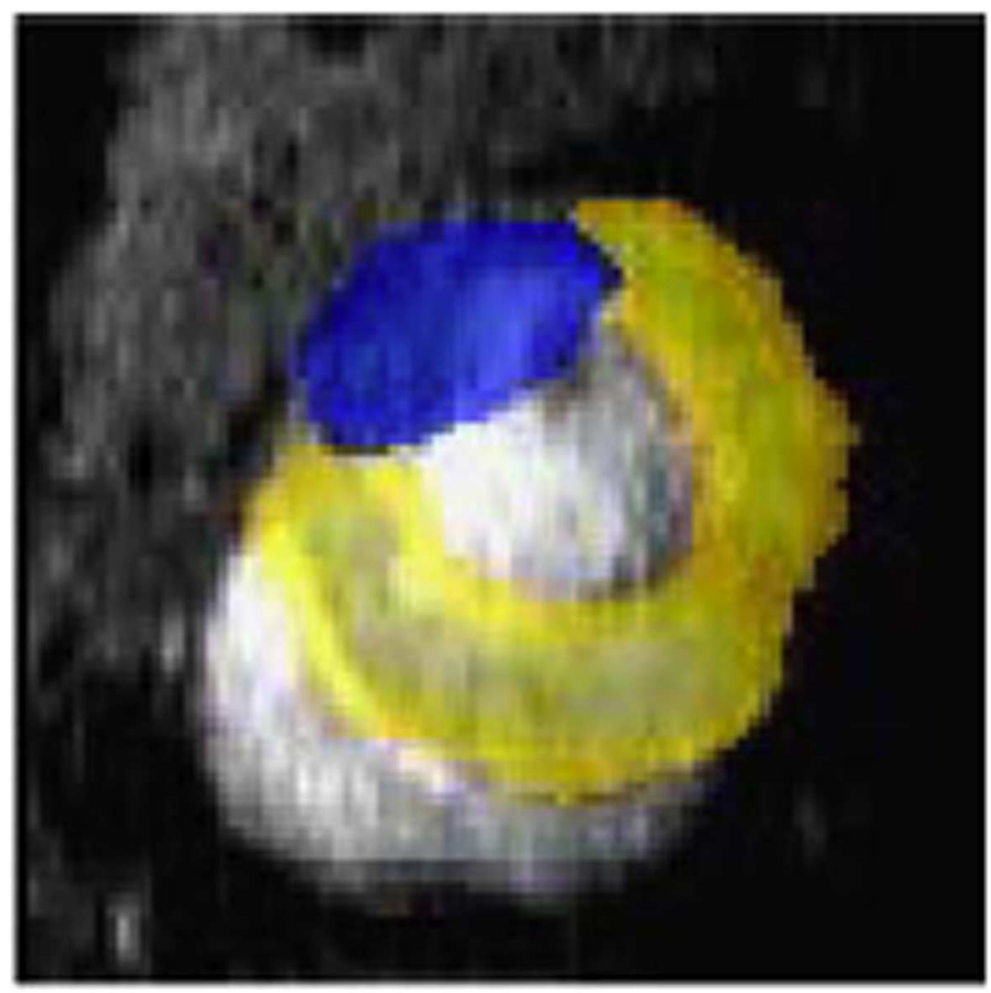

Fig. 7. Coregistered SPECT/SPECT/MRI imaging MRI provides anatomical mapping (grayscale) against ${ }^{111}$ In-oxyquinoline labeled stem cells (b/ue) grafted to infarcted rat myocardium and simultaneously acquired ${ }^{99}$ Tc-sestamibi maps (yellow) of perfusion deficient regions. Reproduced with permission from [49].

eliminated these errors and highlights the benefits for a combined instrument that would allow simultaneous multimodality acquisition.

\section{Multimodality Contrast Agents}

In the papers described up to this point, multimodal imaging was achieved by using separate contrast agents for each modality. The reports of multimodal, integrated contrast agents that are detectable by more than one modality is a much more recent field, and by far the largest number of reports are for single agents combining optically detectable species with MRI detectable species. These agents are paramagnetic (or ferromagnetic) and luminescent with widely varying structures and composition. Simple constructions of polymers or dendrimers coupled to Gd and fluorescent dyes have been reported [51], [52], as have more synthetically challenging combinations such as a small molecule Gd-rhodamine fusion structure [51]. A very popular construct is to conjugate luminescent quantum dots to other contrast agents. Gd coupled to the surface of luminescence quantum dots [53] or superparamagnetic iron oxide particles (SPIO) coupled to fluorescence dyes such as Cy5.5 have been reported by many groups [54]-[58]. Another system, similar to the commonly used secondary antibody amplification techniques used in histology, employs a magnetically labeled antibody in conjunction with a secondary antibody labeled with a fluorescent dye [59].

More recently, efforts have been directed at fusing different types of contrast agents. For example, SPIO has been coated with a luminescent CdSe [60], or Yb or Er [61], or silica dye infused layer [62]. Exotic heterostructures have been reported containing combinations such as a quantum dot with an FePt particle [63] or an FePt and Au particle fusion [64]. In some of our work, we have reported Fe-Au nanoparticles. The gold provides colorimetric bright field contrast. One very complex design includes multiple layers consisting of a silica core containing luminescent $\mathrm{Ru}($ by) coated with a Gd containing layer and an outer functionalized silica layer [65]. Other work in our lab has focused on developing quantum dots in which the paramagnetism is an integral part of the quantum dot, and we have recently reported development of $\mathrm{Mn}$-doped CdSe-type quantum dots and Si quantum dots. In recent work, we describe the synthesis of core/shell CdSe/ $\mathrm{Zn}_{\mathrm{x}} \mathrm{Mn}_{1-\mathrm{x}} \mathrm{S}$ nanoparticles [60]. As shown in Fig. 8, cells incubated with the nanoparticles could be imaged by fluorescence microscopy and MRI. Fig. 8(a) shows cells incubated with the dual-mode quantum dots for one hour after washing and imaging by confocal microscopy. Internalized quantum dots are clearly luminescing in the cytosol. The same cells were then lysed and placed into tubes for MR imaging, shown in Fig. 8(b). Cells 


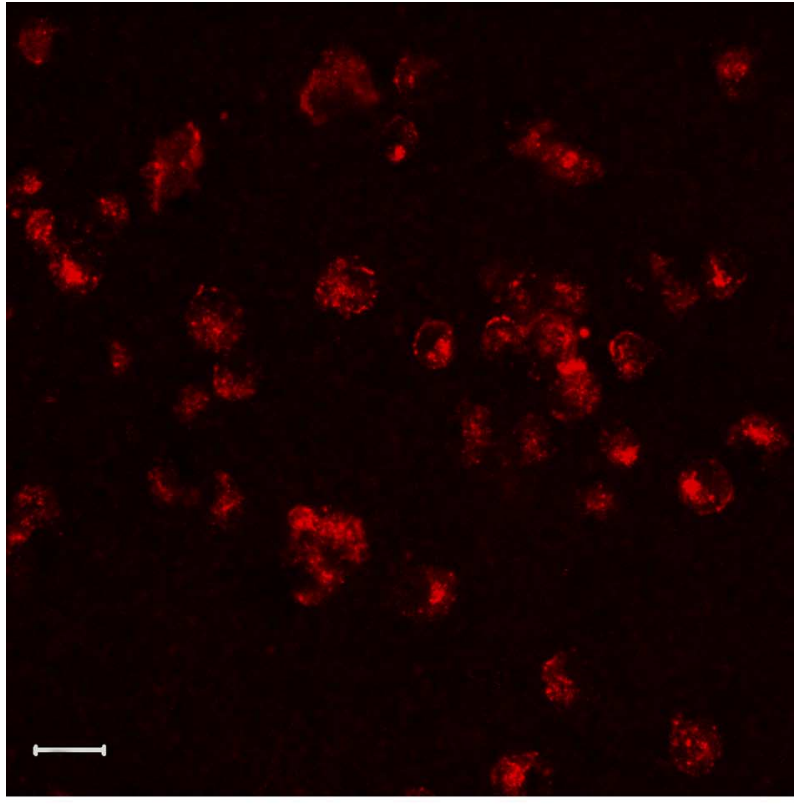

(a)

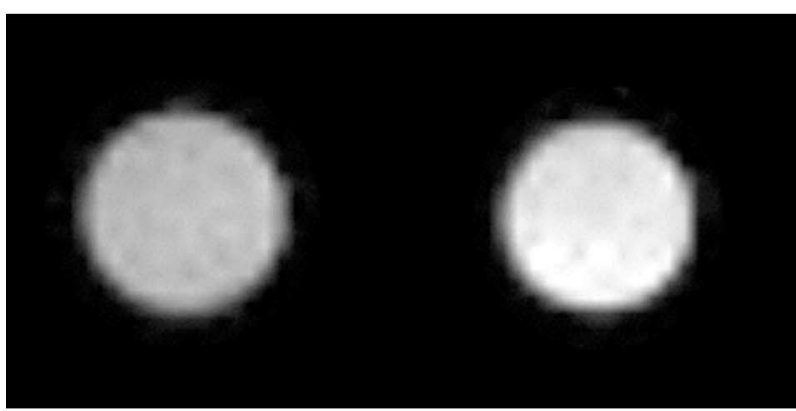

(b)

Fig. 8. MRI of quantum dots in cells. (a) Cells internalize sufficient multimodal quantum dots for detection by confocal microscopy. Scale bar $=\mathbf{2 0}=\mathbf{2 0} \mu \mathrm{m}$. (b) Internalized quantum dots produce MRI contrast. T1-weighted images from tubes containing cell lysates. Reproduced with permission from [60].

on the right show increased contrast after uptake of the quantum dots.

There are very few examples of radio-labeled, multimodal imaging agents in the literature, but there is strong interest in development of these types of tools, particularly as multimodality imaging systems become more widespread. The increasing interest in multimodal imaging has led to development of systems to simplify the synthesis and purification of multimodal agents, although strictly speaking these are not necessary for all-in-one agents. For example, Humblet et al. report an high performance liquid chromatography (HPLC) system with multiple detection elements for purification of agents for MRI, SPECT, PET, radiotherapy or near-infrared optical imaging [66]. They use an agent targeted to prostate-specific membrane antigen coupled to $1,4,7,10$-tetraazacyclododecane-N,
$\mathrm{N}^{\prime}, \mathrm{N}^{\prime \prime}, \mathrm{N}^{\prime \prime \prime}$-tetraacetic acid (DOTA) as an example. The DOTA can hold $\mathrm{Gd}^{3+}$ for MRI, ${ }^{111} \mathrm{In},{ }^{185} \mathrm{Re}$, or ${ }^{99} \mathrm{~m}_{\mathrm{Tc}}$ for SPECT, or $\mathrm{Y}^{3+}$ for therapy. In their example, each molecule holds only one DOTA and therefore is suited for only one modality for imaging. But the idea of the HPLC system is that it is capable of purifying any of the derivatives. This is accomplished by connecting a nonradioactive subsystem, containing separate mass spectrometry, absorbance, and fluorescence detectors, to a radioactive subsystem with beta and gamma detectors contained in a hot cell. Given the great variation in sensitivities of the clinical imaging modalities, it could be useful in certain applications to use variants of the same imaging agent. However, there is no guarantee that the signal will be coming from the same location in coregistered images. In addition, the variants will all compete for binding to the same target, and because the lower sensitivity agents must be given in higher doses, this may be an issue for labeling with the higher sensitivity agents if they are delivered in a mixture. For these reasons and others, it can be beneficial to have an agent carrying multiple labels on a single molecule or particle.

An example of a true all-in-one multimodal agent describes a single agent consisting of an Arginine-GlycineAspartic Acid (RGD) peptide coupled to ${ }^{111}$ InDTPA and an infrared (IR) dye that is targeted to integrin $\alpha_{\nu} \beta_{3}$ receptors [67]. The peptide ${ }^{111}$ InDTPA and near-IR (NIR) dye were joined as shown in Fig. 9 and used to image human melanoma xenografts in a nude mouse model. Fig. 10 shows the bright light, gamma, and luminescence images from these mice. Fig. 10 shows images from mice after (top) injections with the compound only or (bottom) preinjection of RGD peptides followed by the compound one hour later. In the image, the arrowhead refers to a tumor in the hind leg generated from M21 while the arrow

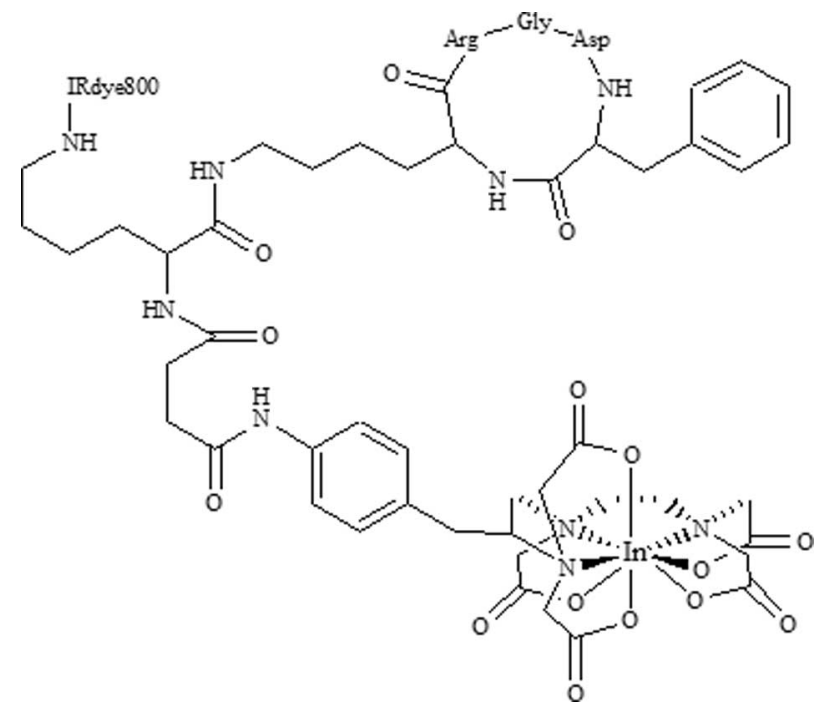

Fig. 9. RGD peptide coupled to ${ }^{111}$ InDTPA and an IR dye. Reproduced with permission from [67]. 


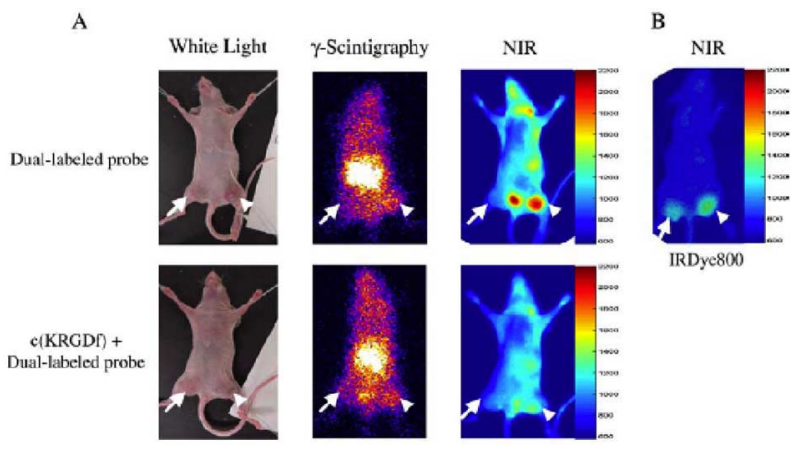

Fig. 10. NIR and SPECT images from human melanoma xenografts in a rat model using the RGD-111 InDTPA-IR dye construct. Reproduced with permission from [67].

indicates a tumor arising from M21-L, which is negative for the integrin receptor being targeted. The M2-L tumors do not show enhancement by either modality, while the M21 tumors show enhancement by both NIR and $\gamma$-scintigraphy. Preincubation with the peptide, partially blocked uptake of the targeted agent. Fig. 10(b) shows an animal injected with the NIR dye alone. The authors say that the level of uptake is similar to mice that received the compound in the presence of blocking agents. In their discussion, the authors envision using this dual-mode probe to first validate use of NIR probes in the clinic, and then in the ultimate clinical application, the radiotracer will be used to screen patients for cancerous lesions. Then, over time, after the radiation has decayed, the optical part of the probe could be used to guide subsequent biopsies or other surgical procedures.

In work recently reported by our group, we have generated gadolinium-based MRI contrast agents and iron oxide-based probes that can be labeled with ${ }^{64} \mathrm{Cu}$ for PET imaging [68], [69]. These agents have potential for dualmode PET/MRI imaging of vascular inflammation. These are targeted molecular imaging agents that will enable in vivo assessment of macrophage accumulation associated with cardiovascular disease. Macrophages express a number of cell surface receptors, including class A types I and II (SR-AI and II) and CD36 [70]-[72]. We target the probes to macrophages through scavenger receptor type A (SR-A) expressed by macrophages. We have selected SR-A as a target as these receptors are expressed primarily by macrophages; have not been found in normal aortic endothelium; and are found in atherosclerotic lesions at all stages [73]-[75]. The overall concept is to assess plaque burden and vulnerability via multimodal imaging methods, using the sensitivity of PET to identify putative lesions and the resolution capability of MRI to assess plaque morphology and macrophage density/distribution in plaques. PET has the potential to survey large tissue volumes and detect small but significant lesions; however, PET lacks the resolution to fully visualize lesion structure. MRI has the resolution to image vessel walls and report on plaque composition but lacks the sensitivity to survey large volumes for small lesions. MRI studies of carotid arteries show promising results that support the feasibility of realtime intravascular imaging but contrast-to-noise must be improved before clinical use is possible. Contrast enhancement can be achieved through the use of exogenous agents. Combining the high sensitivity of PET with the high resolution of MRI through the use of a dual-mode agent has the potential to enable both detection and characterization of lesions in a single study.

\section{Design of Dual-Mode PET/MRI Agents}

We have designed two basic types of agents that are either 1) polymer- or 2) nanoparticle-based. The basic composition of both types of agents is a fusion of three components - a targeting component, a paramagnetic component that can be detected by MRI, and a positronemitting component for PET. In order to increase options for MR imaging parameters, we have incorporated diverse approaches to the problem-different types of ligands (protein, polynucleotide, and polysaccharide) with different magnetic contrast mechanisms $\left(T_{1}\right.$ versus $T_{2}$ ) were generated. In the polymer agent, as shown in Fig. 11, the polymer is the targeting component and consists of amino acid or nucleotide polymers (mal-BSA, polyI, or polyG) that are ligands recognized by macrophage scavenger receptors. The polymer is conjugated to chelators that can hold either paramagnetic gadolinium or ${ }^{64} \mathrm{Cu}$. The chelator selected, DOTA binds strongly to either metal [76], [77]. The nanoparticle agent in Fig. 12 consists of a paramagnetic

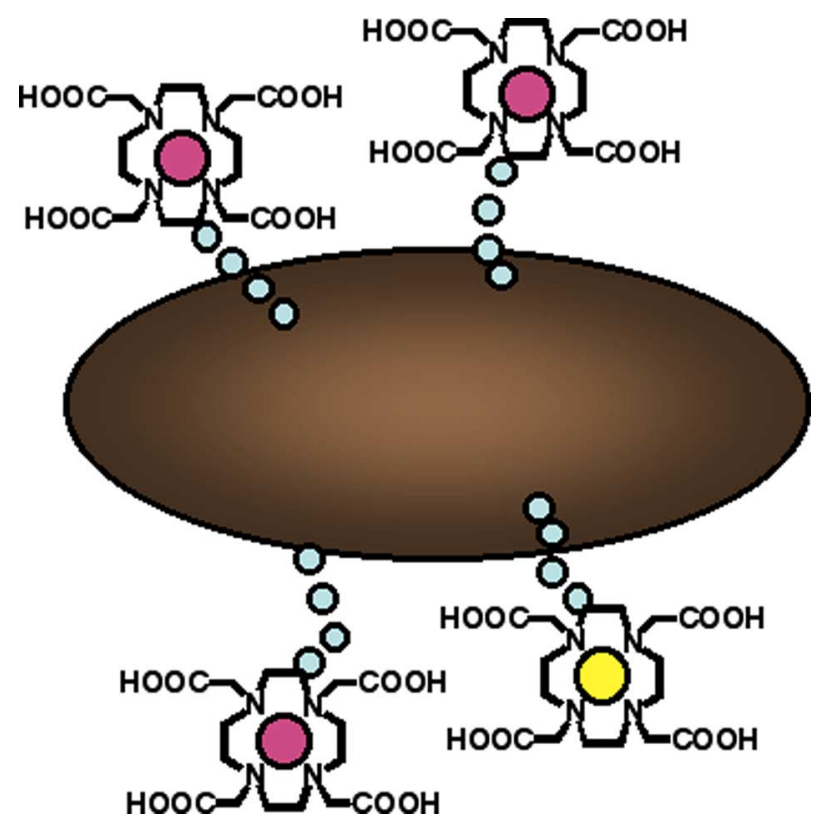

Fig. 11. Schematic of mal-BSA based dual-mode agent. Gd (pink), Cu-64 (yellow). 


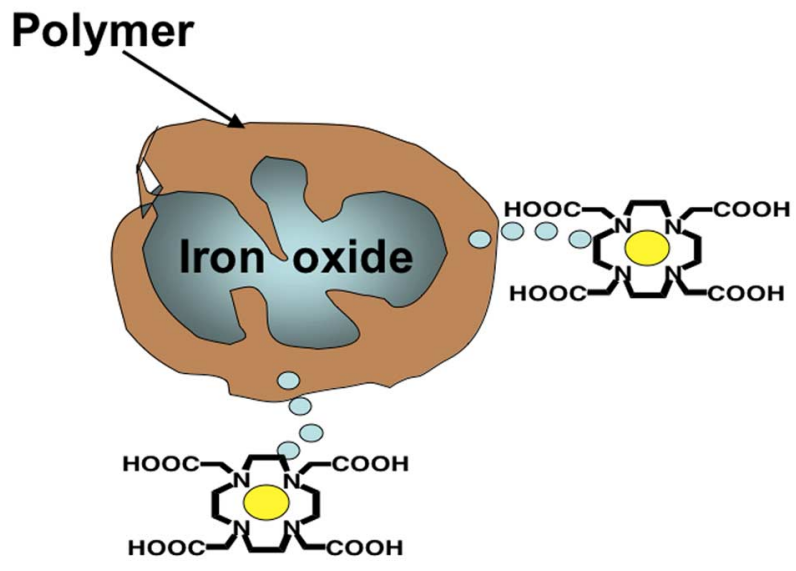

Fig. 12. Design of dual-mode dextran sulfate coated nanoparticle.

core of iron oxide that is coated by a layer of the targeting polymer. The targeting polymer is a polysaccharide (dextran sulfate) conjugated to DOTA chelating ${ }^{64} \mathrm{Cu}$.

The two types of agents differ in their mechanism of MRI contrast. The polymer-based agents containing gadolinium affect $\mathrm{T}_{1}$ relaxation times. $\mathrm{T}_{1}$ agents, such as gadolinium, interact directly with water protons to shorten $\mathrm{T}_{1}$, which results in an increase in signal intensity [78], [79]. The nanoparticle-based agents containing iron oxides affect $\mathrm{T}_{2}$ relaxation times. $T_{2}$ agents shorten $T_{2}$ by a through-space, magnetic field effect, resulting in a decrease in signal intensity. Both methods enhance contrast by altering the signal intensity of labeled tissue relative to neighboring tissues. $\mathrm{T}_{2}$ contrast agents are generally more sensitive and produce effects at lower concentrations, while $\mathrm{T}_{1}$ contrast agents are capable of greater dynamic range because they produce positive contrast. Different contrast mechanisms may show greater utility for particular types of imaging sequences or tissue environments. By synthesizing both types of probes, we have the greatest versatility to affect image contrast in any system. Furthermore, the modular nature of the probes allows us to expand to other imaging contrast mechanisms, such as fluorescence, by attaching additional moieties.

\section{E. Biomolecule-Based Probes}

1) Targeting Macrophages via Scavenger Receptor SR-A: We have previously shown that restenotic plaques can be specifically labeled using a ligand to the macrophage scavenger receptor, namely, class A type scavengers (SR-A) [80]. In these studies, a ligand to SR-A, maleylated bovine serum albumin (mal-BSA), was labeled with a fluorescent marker (Texas Red) and found to be specifically taken up by cells in culture. Labeled mal-BSA was taken up by macrophages but not by smooth muscle cells or endothelial cells. This uptake was inhibited by excess unlabeled mal-BSA, which supports a receptor-mediated mechanism (not shown).
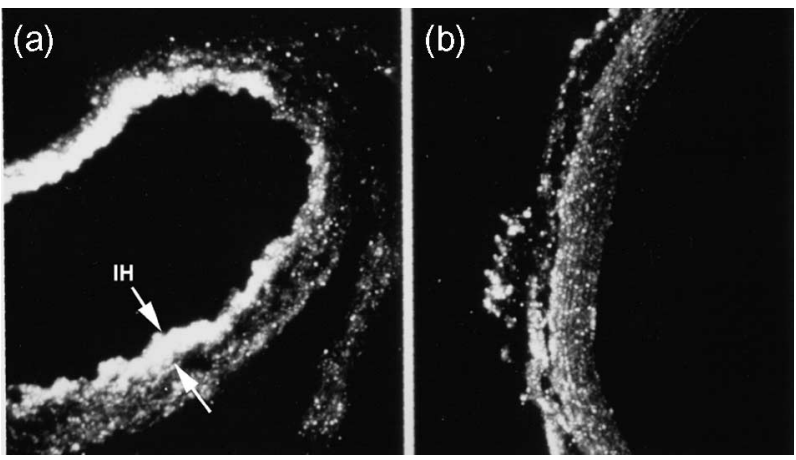

Fig. 13. Fluorescent labeling of injured arteries. Injured arteries (a) are labeled with Texas Red mal-BSA conjugates while (b) uninjured arteries are not.

Further studies in the rat model showed that restenoses occurring after balloon catheter deendothelialization were brightly labeled by the fluorescent Texas Red mal-BSA conjugates. In these investigations, abdominal aortas in rats were denuded of intima by pulling an inflated balloon catheter through three times. Two weeks after injury, animals were injected with labeled mal-BSA or labeled BSA as negative control. Four hours after administration of the drug, abdominal aorta and uninjured thoracic aorta controls were collected and prepared for cryohistology. Frozen tissue sections were observed by fluorescence microscopy. Fig. 13 shows (a) a labeling of the intimal hyperplasia $(\mathrm{IH})$ in an injured abdominal artery (b) that is not observed in uninjured thoracic artery controls. Additional controls, shown in Fig. 14, demonstrate that labeling of IH occurs only (c) with mal-BSA and (a) not with free dye alone or (b) labeled BSA.

These results suggest that ligands of SR-A can be used as a delivery vehicle to shuttle labels to restenosis and that perhaps other ligands or labels can be targeted to
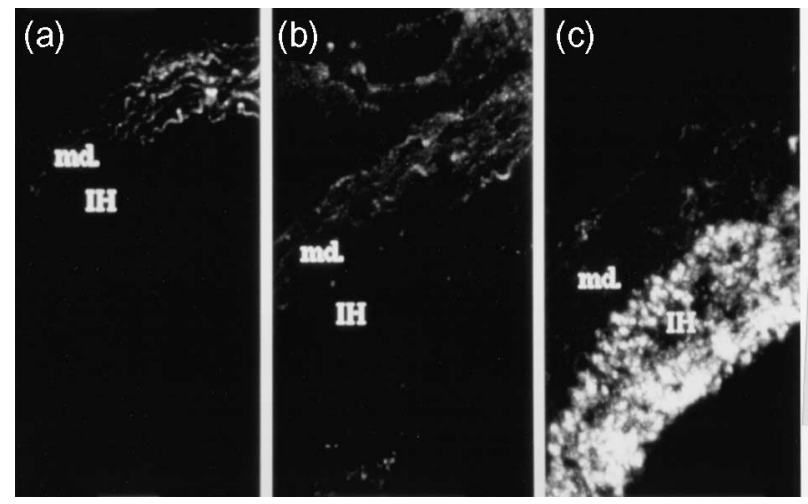

Fig. 14. Specific labeling of intimal hyperplasia. Injured arteries were exposed to (a) Texas Red alone, (b) Texas Red-BSA conjugate, and (c) Texas Red mal-BSA conjugate. 
restenoses in a similar manner. We have used two different ligands of SR-A to localize dual-mode contrast agents to restenoses for the purpose of noninvasive imaging of plaques.

2) Mal-BSA Dual Mode PET/MRI Probe Synthesis: We have synthesized and characterized a biomolecule-based agent using the ligand maleylated bovine serum albumin (mal-BSA) [68]. As illustrated earlier, this agent is composed of mal-BSA conjugated to chelated $\mathrm{Gd}^{3+}$, paramagnetic ion for MRI contrast, and the radionuclide ${ }^{64} \mathrm{Cu}$ for PET. We have demonstrated specific targeting of this agent to macrophages in culture and performed preliminary evaluations of the toxicity and biodistribution of this agent. Here we summarize the results for this agent.

BSA was maleylated by conjugating maleyl groups to free amines by published methods [81]. Dual-mode agents, detectable by PET and MRI, were generated by first coupling the chelator $p$-isothiocyanatobenzyl-1, 4, 7, 10tetraazacyclo-dodecane 1, 4, 7, 10-tetraacetic acid ( $p$-SCNBz-DOTA) to remaining free lysines and then inserting the metals; fortuitously either metal can be strongly coordinated by this chelator [77], [82], [83]. MRI properties were optimized before introduction of the radionuclide. $\mathrm{T}_{1}$ relaxivities for the mal-BSA based agents were comparable to or better than the literature reported and commercial agents.

3) Targeting of Mal-BSA Probe to Macrophages In Vitro: The recognition and uptake of the probes by their target macrophages were tested on cells in culture. To confirm that uptake was specific, macrophages were incubated with increasing concentrations of the mal-BSA based agent, or a matched control agent that contained the same number of Gd-DOTA groups but lacked the maleyl groups required for recognition by the scavenger receptor. In Fig. 15, the top row shows data from cells incubated with mal-BSA(Gd-DOTA) 22 , the middle row contains BSA $(\text { Gd-DOTA })_{22}$, and the bottom row is a pure water control. As evident in the figure, there is no uptake of the labeled BSA probe, while there is increasing signal intensity with increasing concentrations of the mal-BSA agent as expected.

In order to verify receptor-mediated uptake, cells were incubated with a fixed concentration of labeled contrast agent, 100 micromolar mal-BSA(Gd-DOTA $)_{15}$, and increasing excess of a matched unlabeled (no Gd) contrast agent. In Fig. 16, we see that contrast enhancement is reduced with increasing excess of competing unlabeled contrast agent (from left to right, 0, 0.01, 0.1, 1, $2 \mathrm{mM}$ competitor, water blank). The numbers below each image give the $T_{1}$ value for the samples shown and averaged over triplicate samples. Uptake that can be competed away by unlabeled ligands is indicative of receptor-mediated uptake. These studies demonstrated

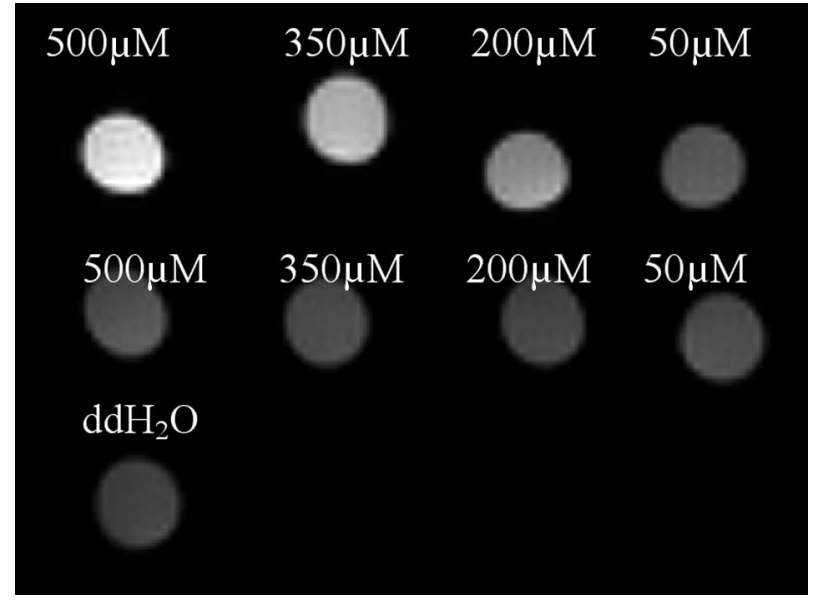

Fig. 15. Uptake of macrophage targeted probe is specific. Cells were incubated with (top) mal-BSA agent or (middle) Gd-DOTA matched BSA control.

that the agent can label macrophages for MRI contrast at reasonable concentrations. Furthermore, cells were imaged at densities of $3 \times 10^{5}-2 \times 10^{6} \mathrm{cells} / \mathrm{ml}$; this is on the order of mean macrophage densities observed in average human postmortem type IV-VI plaques $\left(\sim 3.5 \times 10^{4}\right.$ macrophage/ml [85]).

For PET imaging, these mal-BSA agents were coupled to ${ }^{64} \mathrm{Cu}$ DOTA. In normal rats, the contrast agent introduced by tail vein injections localized primarily in the liver, kidneys, and spleen is as shown in Fig. 17. In preliminary experiments in the balloon-injured rat model, we found accumulation of contrast agent in the injured vessel. Fig. 18 shows the PET signal from the mal-BSA probe accumulated in an injured thoracic artery (upper arrow). The spine and ribs (lower arrow) are clearly visible in a serially acquired CT image that is coregistered with the PET for anatomical reference. We have also been able to image vessels walls by MRI (Fig. 19). These preliminary images illustrate the promise for these dual-mode agents to visualize macrophage accumulations in atherosclerotic plaques.

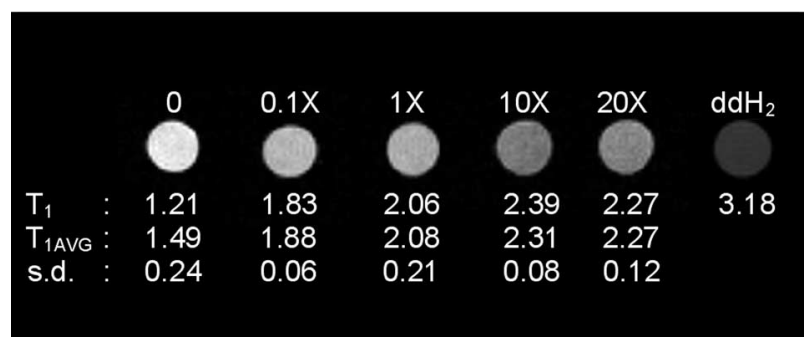

Fig. 16. Competition studies to verify specificity of uptake. Cells were incubated with mal-BSA agent in presence of increasing amounts of competing unlabeled mal-BSA. 


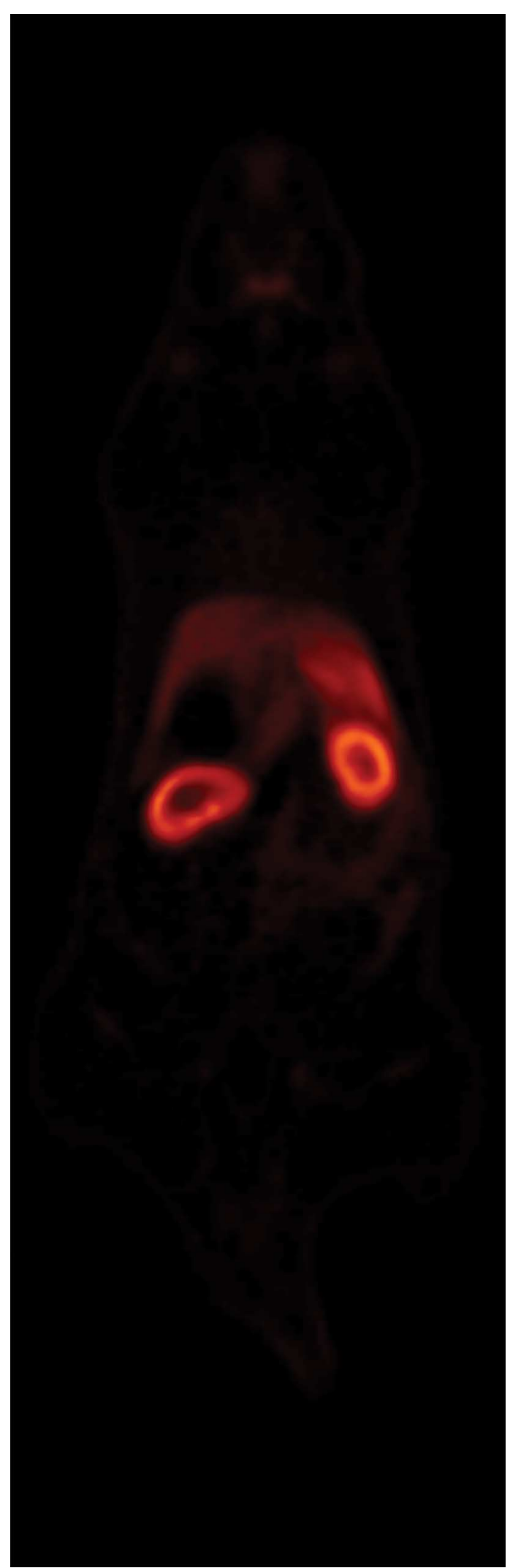

Fig. 17. Biodistribution of GdDOTA- ${ }^{64}$ CuDOTA-mal-BSA $4 \mathrm{~h}$ after tail-vein injection to normal rats localized primarily to liver, kidney, and spleen.

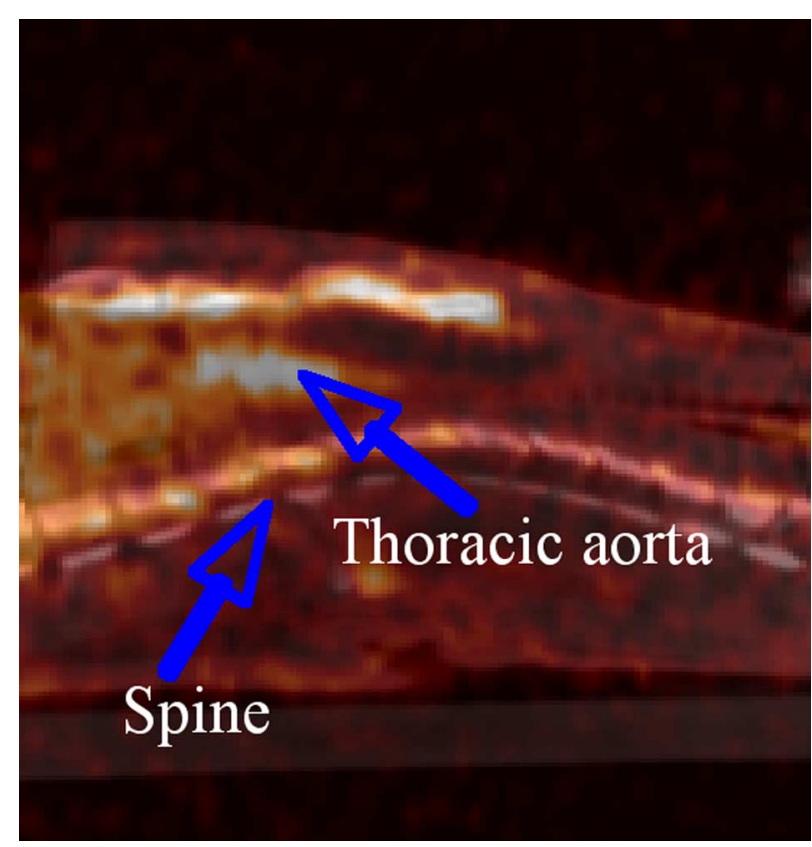

Fig. 18. Thoracic aorta of rat damaged using balloon embolectomy catheter through the carotid artery, imaged using PET/CT.

\section{F. Nanoparticle Based Probes}

1) Nanoparticle Synthesis: The designed dual-mode nanoparticle-based probes consist of a core of iron oxide coated with the scavenger receptor ligand dextran sulfate. Iron oxide serves as the $\mathrm{T}_{2} \mathrm{MRI}$ contrast agent, and for PET, ${ }^{64} \mathrm{Cu}$ (DOTA) is incorporated by conjugation to the dextran sulfate surface. This is a one pot synthesis in which $\mathrm{Fe}^{3+}$ and $\mathrm{Fe}^{2+}$ are mixed with reduced polysaccharide in

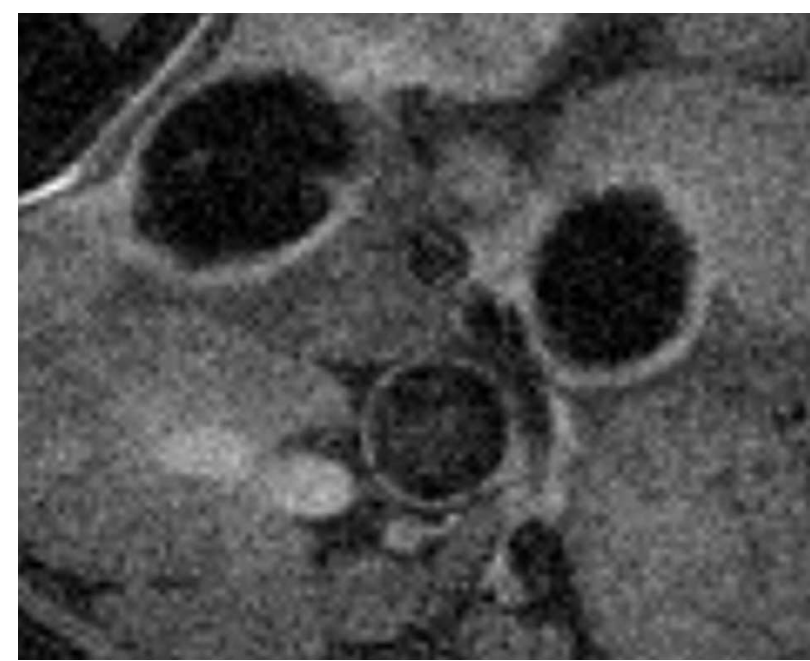

Fig. 19. Abdominal aorta $T_{1}$-weighted MR image with cardiac gating. Additional respiratory gating will improve image sharpness. The abdominal/thoracic aorta is the smaller vessel in the center. 


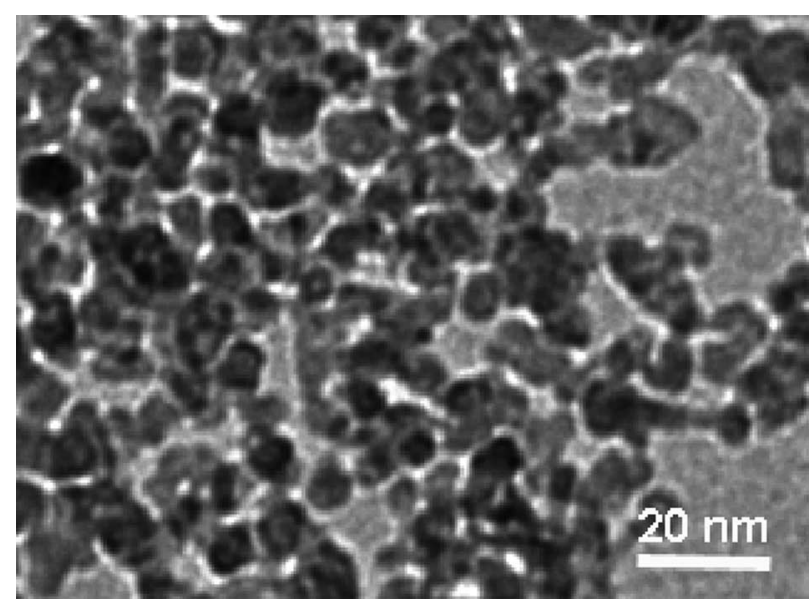

Fig. 20. TEM image of ADIO. Dextran sulfate coated iron oxide nanoparticles have cores of $\sim 5.2 \mathrm{~nm}$ as determined by TEM.

the presence of base at low temperature, then gradually heated and the particles spontaneously assemble. Briefly, dextran sulfate coated particles were synthesized by doping a small amount of dextran sulfate into a dextran solution in the presence of iron chloride salts. More detailed syntheses and modifications to the basic protocol are described in our recent work on size-controlled synthesis of these nanoparticles [69]. The relaxivity values for the $5 \mathrm{wt} \%$ dextran sulfate doped particles [anionic dextran iron oxide (ADIO)] were comparable to a literature dextran coated iron oxide preparation (SPIO) at $7 \mathrm{~T}$ [86]. Structural analysis by transmission electron microscope (TEM) (Fig. 20) shows iron oxide cores of $5.2 \pm 1.3 \mathrm{~nm}$ in diameter. Overall hydrodynamic radius determined by dynamic light scattering was $88.5 \mathrm{~nm}$, suggesting multiple cores per nanoparticle.

2) Targeting of Nanoparticle-Based Probes to Macrophage In Vitro: The effect of dextran sulfate incorporation on cellular uptake was explored using P388D1 macrophages. Uptake of the ADIO particles by macrophages is greater than uptake of plain SPIO. Dextran coated nanoparticles can be nonspecifically phagocytosed by macrophages, but receptor targeted dextran sulfate coating was anticipated to increase iron uptake efficiency by macrophages. Receptor mediated uptake is a more efficient process than nonspecific pino- or phagocytosis. As shown in Fig. 21, the $\mathrm{T}_{2}$ values for cells incubated with nanoparticles in iron concentrations of $100 \mu \mathrm{g} / \mathrm{mL}$ and greater are significantly lower for the ADIO particles (blue points). Images of suspensions of these cells by MRI, as shown in Fig. 22, show greater decrease in signal with concentration for the ADIO samples (top row) compared to the SPIO samples (bottom row). This suggests that incorporation of sulfate groups into the particle coating results in increased uptake of iron oxide compared to dextran coating alone.

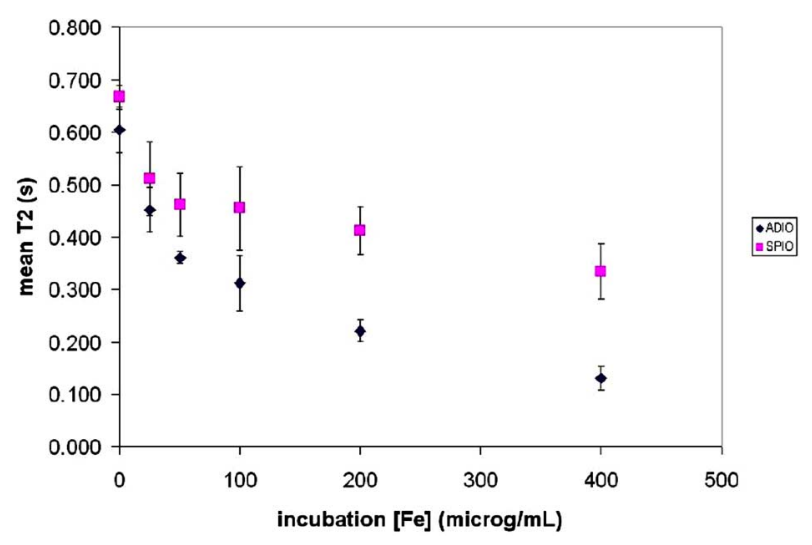

Fig. 21. Cells incubated with targeted dual-mode agent show greater decrease in $\mathrm{T}_{2}$ than cells incubated with nontargeted nanoparticles.

Labeling of the nanoparticles with positron-emitting ions is achieved by coupling p-SCN-DOTA to the nanoparticles through amine groups and inserting ${ }^{64} \mathrm{Cu}$.

One may surmise that reported multimodal agents only hint at the future of the field, and MRI/optical agents have had much more attention in recent literature. The development of multimodal agents for combined PET/MR imaging is still in its infancy, and there is still some debate on the need for putting detectability by both modalities on a single probe. When fused instruments were proposed, these too were met with resistance. But just as the benefits of fused instruments have revealed themselves, multimodal agents have a place in the diagnostic imaging repertoire. One can envision their requirement in applications needing precise confirmation that the signal detected by one modality is from the same cellular or tissue source as the signal detected in another, such as for guided biopsies. And as mentioned earlier, the
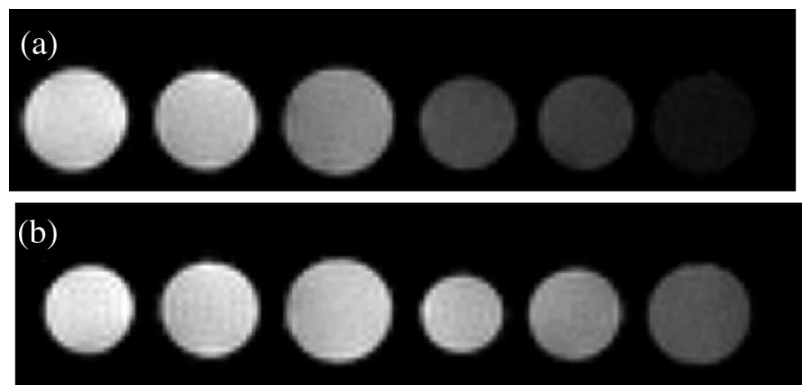

Fig. 22. $T_{2}$ weighted images of cell lysate suspensions for (a) ADIO and (b) SPIO. For both (a) and (b), from left to right, control, $25,50,100,200$, and $400 \mu \mathrm{g} \mathrm{Fe} / \mathrm{mL}$. A significant decrease in signal intensity for ADIO samples compared to SPIO samples is seen. The images are from $\mathrm{TE}=\mathbf{3 7 5} \mathrm{ms}$ of a multiecho spin echo sequence. Parameters were $T R=3200$ ms, 75 ms echo spacing, FOV $=5.5 \mathrm{~cm}$, matrix size $128 \times 128$, and slice thickness $1.2 \mathrm{~mm}$. The difference in diameter of the samples is due to the image plane passing through different sections of the conical tubes. 
use of the differentially labeled agents with the same molecule carrier can create an issue with competition by the agent delivered in higher doses. Given the great difference in sensitivity between PET and MRI, the multimodal PET/MRI agent will essentially be a mixture of dual-labeled agents and those with just MRI detectability. But the added assurance that the signal from both modalities comes from the same molecule can be advantageous.

\section{APPLICATIONS AND SPECULATIONS}

As outlined in the introduction, the basic physical underpinnings of PET and MRI are qualitatively different. In PET, the three-dimensional location and concentration of a positron-emitting compound are measured, often over time, and provide the spatial intensity variations that make up the PET image. The concentration of the compound at a particular place at a particular time is a function of the biological process or processes that the compound has been designed to reflect (e.g., FDG reflects glucose metabolism, FLT reflects cellular proliferation). In the vast majority of MRI scans, the spatial intensity variations that make up the MR image arise from differences in water physicochemical properties and concentration of water protons across the sample. For example, in the brain, white matter, gray matter, ventricles, etc. (i.e., anatomical structures) are distinguishable in an MR image because the specific tissue characteristics perturb the local water protons in different ways, giving rise to local differences in water $\mathrm{T}_{1}, \mathrm{~T}_{2}$ and/or density. Disease manifestations, such as tumors and plaques, modulate tissue properties (e.g., permeability, viscosity, cellularity, diffusion, temperature, heterogeneity) and change MR characteristics (e.g., $\mathrm{T}_{1}, \mathrm{~T}_{2}$ ), making them visible in an MR image. As discussed above, contrast agents play much the same role in MRI as radionuclide labeled compounds in PET imaging, although unlike PET radiotracers, they are not observed directly but through their influence on nearby water protons. It is the interplay between the direct and the indirect, between the particular and the general, that underlies both the utility and the fascination of simultaneous PET/MR imaging. In this section, we outline some of the more obvious utilities of simultaneous PET/MRI that have been confirmed in our preliminary studies and detail speculations and expectations in three particular applications that will serve as exemplars of how this technology may be applied in future studies in a wide variety of situations: imaging of brain function, mouse phenotyping, and imaging of tumor biology.

\section{A. Obvious Benefits}

The most immediate benefit of the melded system is to provide accurate and precise high-resolution anatomical context for PET studies. The MRI and PET images are "automatically" in register because they are recorded at the same time on the same sample with no sample movement (other than that due to physiology). This may seem rather trivial but, as discussed previously, image registration for parts of a specimen that are not malleable (e.g., brain) is not always straightforward, and for malleable tissues is often very difficult. Fig. 23 shows ${ }^{18} \mathrm{~F}^{-}$uptake into the bones of the skull, mouth, and jaw of a mouse by PET, with no uptake into the brain or other surrounding soft tissue. Although in this example uptake in one tissue (bone) and not the adjacent tissue (brain) is obvious, this will not always be the case. In experiments aimed at tracking labeled cells (e.g., stem cells) introduced intravenously, it will be crucial to differentiate between cells in the vascular system and those taken up by specific organs (lungs, spleen, bone marrow). In this case, accurate and specific identification of the location of the PET label by MRI will be essential for even rudimentary interpretation of experimental outcome. Fig. 24 illustrates that measures of the time dependence of FDG uptake and simultaneous anatomical MRI are now straightforward with the integrated PET/MRI systems described earlier. Knowledge of the time dependence of the distribution of labeled cells, complexes, and small molecules will be a necessary part of determining their pharmacokinetics. Thus, the ability to accurately coregister PET and MR images without recourse to complex warping methods [87] will be of tremendous importance in putting information from PET scans in anatomical context in both preclinical and clinical arenas.

Magnetic resonance spectroscopy (MRS) allows the in vivo identification of specific chemical compounds. ${ }^{1} \mathrm{H}$ MRS of the brain can be used to detect relative concentrations of specific metabolites (e.g., glutamine, $\mathrm{N}$-acetylaspartate, creatine, choline) as a function of location. Likewise, ${ }^{31} \mathrm{P}$ MRS can be used to detect high-energy phosphate metabolites (e.g., inorganic phosphate, ATP, ADP, AMP). Information about relative amounts of metabolites and changes in them provide insight into physiological changes in the tissues of interest. The use of ${ }^{19} \mathrm{~F}$ MRS to follow the uptake, metabolism, and secretion of fluorinated drugs takes advantage of the relatively high sensitivity of ${ }^{19} \mathrm{~F}$ in the MR experiment and the essential lack of any background signal [88], [89]. Although of lower intrinsic sensitivity, ${ }^{13} \mathrm{C}$ MRS at natural abundance and with ${ }^{13} \mathrm{C}$ labeled compounds has proved useful in studies of metabolic pathways [90]-[92]. It is natural to consider applications of multilabeled ${ }^{18} \mathrm{~F} /{ }^{19} \mathrm{~F}$ or ${ }^{11} \mathrm{C} /{ }^{13} \mathrm{C}$ compounds in which the high sensitivity of detection of PET is combined with the ability of MRS to provide noninvasive chemical analyses [93]. For example, the development/ discovery of "prodrugs" (compounds administered in an inactive form that are subsequently metabolized in vivo into the active therapeutic) will benefit greatly from the ability to identify in vivo the site(s) and metabolic processes that take the prodrug to its active form. Fluoropyrimidines [5-fluorouracil (5FU)] are among the oldest cancer chemotheraputics with several prodrugs in 
(a)

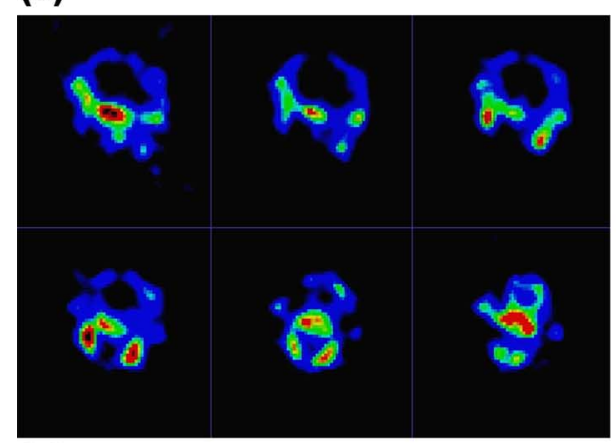

(b)

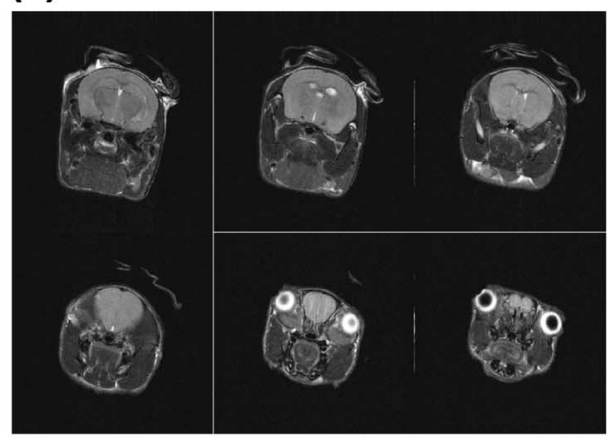

(c)

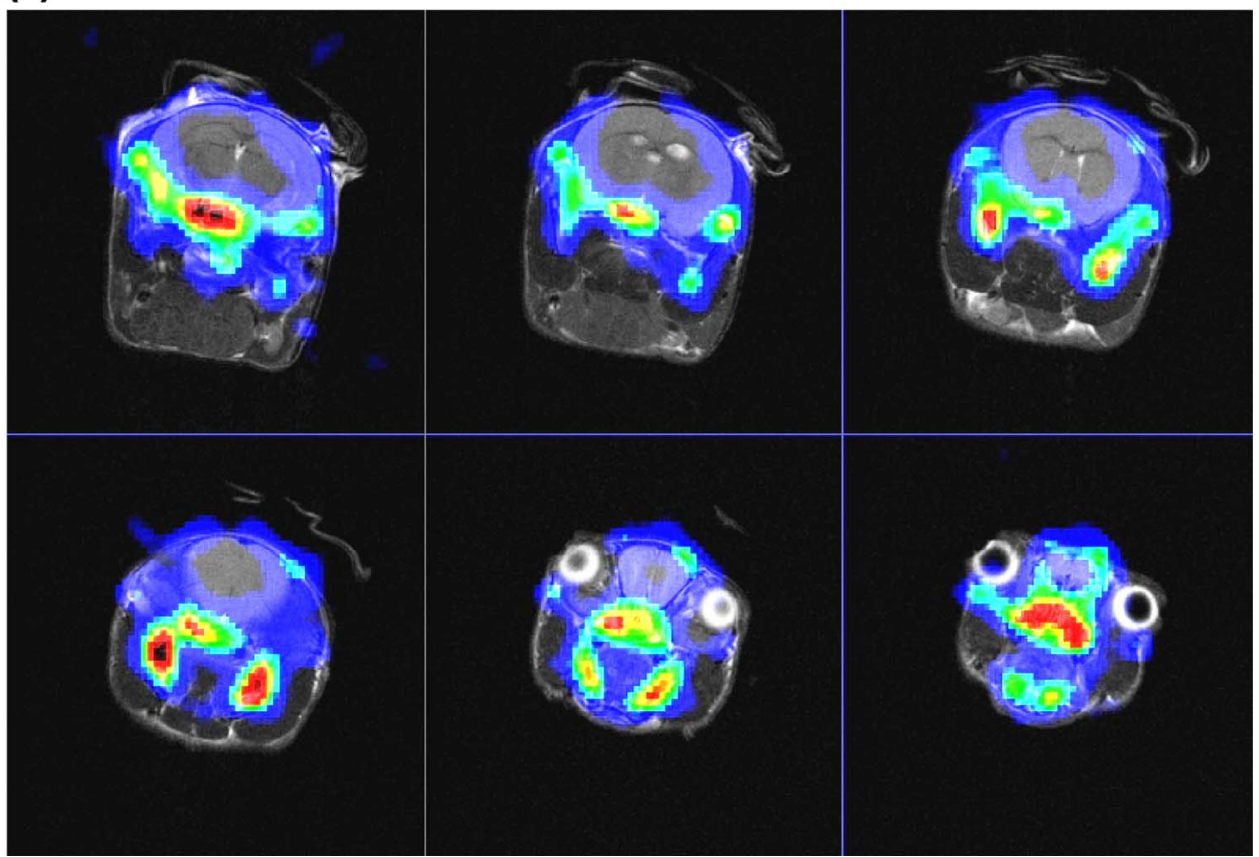

Fig. 23. In vivo simultaneous PET/MRI: (a) PET images showing ${ }^{18} F^{-}$ion uptake; (b) anatomic MR images acquired simultaneously with the PET data; and (c) fused PET and MRI images showing the precise registration of the ${ }^{18} F^{-}$uptake in the bones of the jaw and mouth but not in the adjacent soft tissue. One of the seminal uses of the joint instrumentation will be to unequivocally identify the anatomical locations of the PET radiotracer by the MRI. (Images courtesy of C. Catana, University of California, Davis; and D. Procissi, California Institute of Technology.)

use or development (e.g., capecitabine and gemcitabine). Preclinical PET/MRI studies using ${ }^{18} \mathrm{~F} /{ }^{19} \mathrm{~F}$ prodrugs will point out where, when, and how the prodrugs are metabolized along with determinations of drug efficacy. This information is of critical importance in optimizing prodrug performance. In the clinical setting, joint PET/MRI examination will allow for individually optimized chemotherapy regimens.

The ability to quantitatively image time-dependent changes in gene expression in vivo has significant potential applications in developing and monitoring tissue specific expression of a therapeutic gene. PET reporter gene technologies rely on the binding or intracellular trapping of a radio-labeled moiety [94]-[96]. Substrates useful in other imaging modalites (SPECT, bioluminescence, and fluorescence) may be simultaneously incorporated to make a multimodal reporter gene construct [97], [98]. None of these imaging modalities offers the soft tissue contrast or physiological sensitivity of MRI. Thus, including MR will provide indications of whether the gene expression is actually altering the physiological state of the tissue and supply anatomical context.

\section{B. Brain Function}

There is a long history of using both MRI and PET in brain activation studies in human and small animal model systems [99], [100]. Cerebral blood flow (CBF) can be measured with PET using $\mathrm{H}_{2}^{15} \mathrm{O}$ and with MRI using bolus application of contrast agents [101]. Correlates of neuronal activation are measured with PET by 
mapping glucose metabolism with FDG uptake measurements, while MRI uses vascular response to stimulation evaluated using changes in blood oxygen level dependent contrast (BOLD). Although there have been many studies correlating PET and MRI measures of brain activation [102]-[117], direct simultaneous measurements will provide unequivocal comparisons. PET/MRI small animal studies with simultaneous bolus IV MRI contrast agent and $\mathrm{H}_{2}{ }^{15} \mathrm{O}$ will provide cross-validation of CBF measurements.

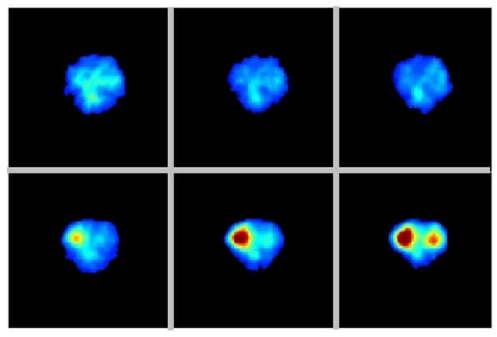

(a)

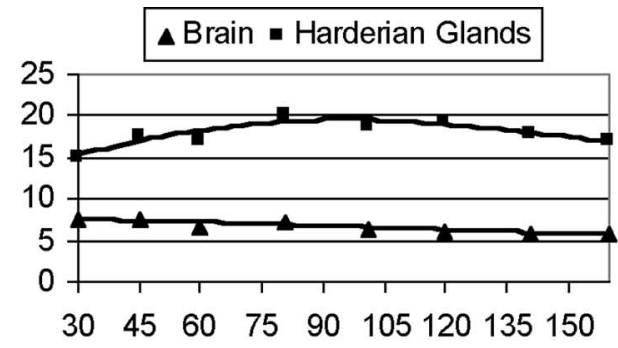

Time (min)

(b)
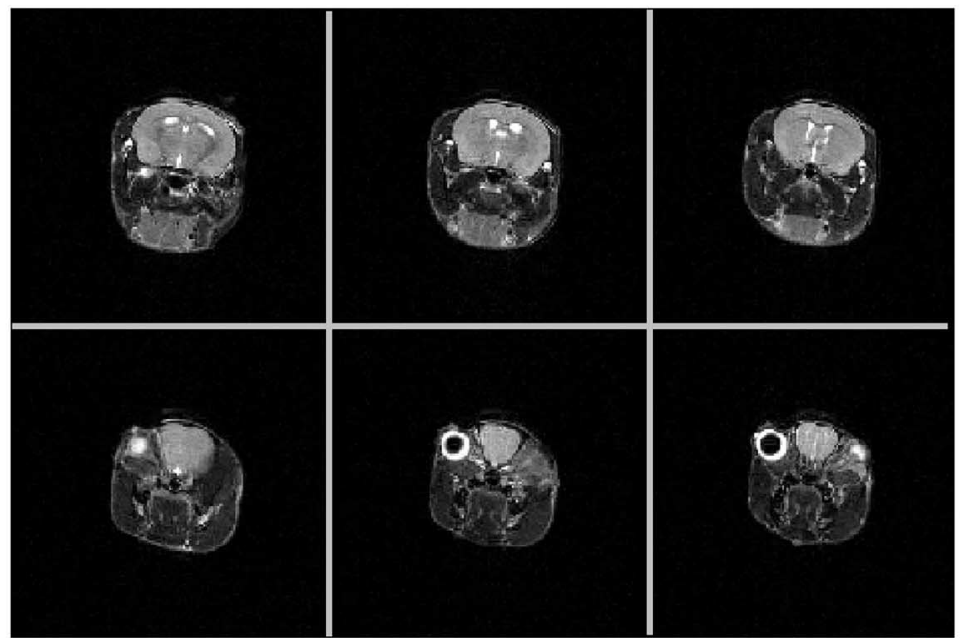

(c)
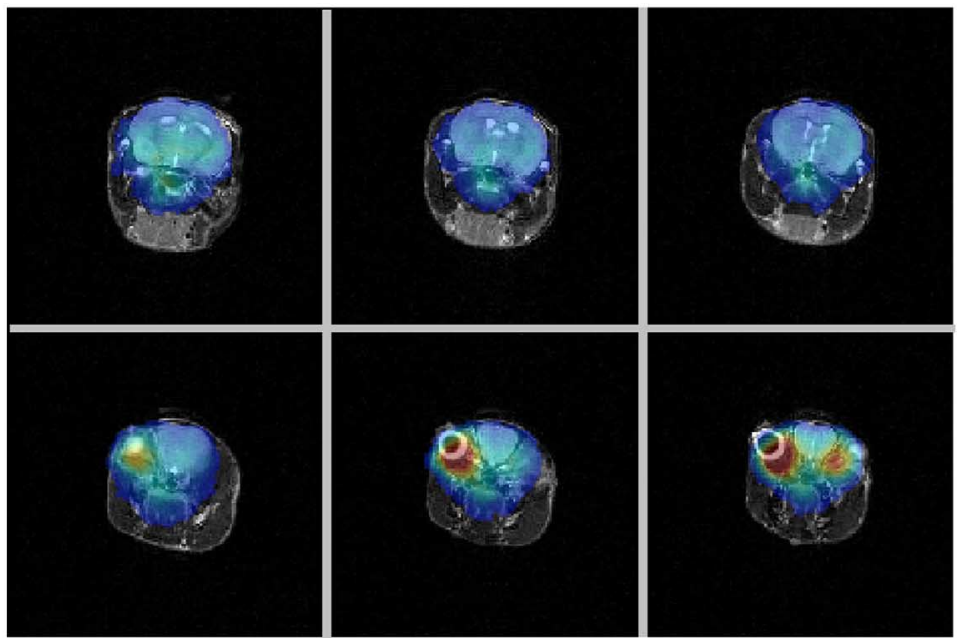

(d)

Fig. 24. In vivo simultaneous PET/MRI: (a) PET images showing FDG uptake in the cortex and Harderian glands; (b) time activity curves obtained from the brain and Harderian glands showing relative uptake of FDG in two different tissues as a function of time after in injection; (c) anatomic MR images acquired simultaneously with the PET data; (d) fused PET and MRI images showing the precise registration of the FDG uptake with anatomic structures. (Images courtesy of C. Catana, University of California, Davis; and D. Procissi, California Institute of Technology.) 


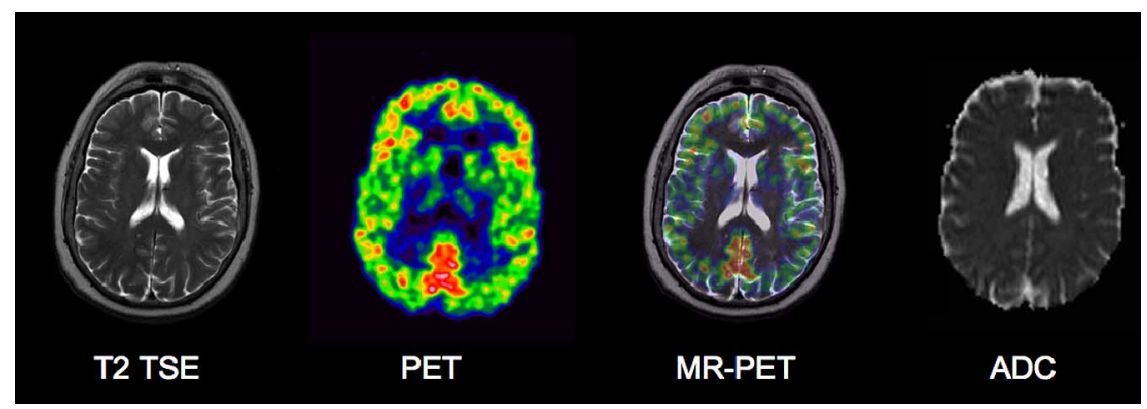

Fig. 25. Simultaneous PET and MR images acquired on a Siemen's Magnetom Trio MRI equipped with BrainPET insert. From left-to-right: $T_{2}$ turbo spin echo (TSE) sequence, FDG PET image, fusion of TSE and PET image, and diffusion EPI sequence. MR and PET data acquired simultaneously. (Courtesy of D. Townsend and C. Nahmias, University of Tennessee; and H-P Schlemmer et al., University of Tübingen.)

Sensitivity is the hallmark of the PET methodology, which has been put to good use in studies of neuroreceptor distribution and activity with radiolabeled ligands, such as ${ }^{11} \mathrm{C}$-raclopride, ${ }^{11} \mathrm{C}$-CFT, and ${ }^{18} \mathrm{~F}$-fluoroDOPA [118]. No similar studies are possible with MRI due to its intrinsically low sensitivity, but high-resolution anatomical MR images provide the context in which to interpret the PET intensity distributions. Simultaneous PET (neuroreceptor and FDG) and FMRI BOLD contrast studies will allow spatial and temporal correlation of biochemical changes with changes in neuronal activation. Merged data will combine the fast time scale and sensitivity of PET with the high spatial resolution of MRI, potentially obviating the need to average across specimens. Fig. 25 shows results from a prototype human PET/MRI system where FDG uptake is shown overlaid on a $T_{2}$ weighted MRI scan acquired simultaneously.

\section{Mouse Phenotyping}

Transgenic rodents are model systems used to address a vast array of questions about biological mechanisms in both normal and disease states. It is becoming increasingly common to use PET and/or MRI to examine the consequences of altering the genome of an animal [119]-[123]. Whole-body rodent imaging allows an assessment of structural and physiological alterations across organ systems in the merged PET/MRI data. Imaging at various time points will allow assessment of when, as well as what, and where phenotypic changes are manifest. Repeated measurements necessitate repeated removal and replacement of the animal in the imaging system. Although images recorded at different times will need to be aligned, employing a joint PET/MRI system completely relieves the additional burden of repeatedly having to realign images from the two modalities. Brain activation measures already discussed will provide detailed information about changes in the central nervous system (CNS). Mouse models of neurodegenerative diseases are especially tempting targets for joint PET/MRI studies, as development of probes and image acquisition methodologies in preclinical work is liable to be directly transferable to the clinic. Tissuespecific metabolic changes can be assessed measuring whole-body distributions of FDG and correlating this with ${ }^{31} \mathrm{P}$ and ${ }^{1} \mathrm{H}$ MR spectroscopic imaging that provides information about high-energy phosphate intermediates (PCr, ATP, ADP, inorganic phosphate) and metabolites that occur in proton spectroscopy at millimolar levels. Likewise, bone disease and formation can be followed using uptake of ionic ${ }^{18} \mathrm{~F}$ [124]-[126], which is correlated to changes in MRI signal intensity [127]. In many situations, phenotypes change with time (e.g., $\beta$-amyloid plaque deposition in Alzheimer's disease model systems) and developmental stage (e.g., CNS myelination). The ability of the PET/MRI systems to repeatedly image the same animal over time is a significant advantage over other methodologies where this is not possible, while simultaneous imaging guarantees image alignment at each time point. Longitudinal evaluation of the same animal greatly facilitates evaluation of disease etiology and progression through characterization of anatomic, physiological, cellular, and genomic changes.

\section{Monitoring Tumor Response}

Evaluation of tumor growth characteristics and response to therapeutics is essential to gaining a better understanding of in vivo tumor biology and the mechanism of action of specific therapeutics. Tumor size, heterogeneity, and rate of growth are obvious measures of importance that can be determined with MRI, while tumor metabolic activity (via FDG uptake) and proliferation (via accumulation of FLT) can be determined with PET. More sophisticated MR methodologies can provide information about tumor vascularity through the use of low molecular weight contrast agents, as well as tumor necrosis, cell density, and water mobility using $\mathrm{T}_{2}$ and apparent diffusion coefficient (ADC) maps. Likewise, information about tumor hypoxia can be obtained with ${ }^{18} \mathrm{~F}$-misonidazole or ${ }^{64} \mathrm{Cu}$-diacetyl-bis[N4-methylthiosemicarbazone] (ATSM) while radionuclide-labeled tumor specific antibodies and peptides are used to detect location(s) of metastasis. 
Although these measurements are informative when coming from separate imaging sessions, measurements in the same animal at the same time allow direct identification of tumor characteristics revealed in MR images with physiological and molecular characteristics revealed in PET images. Registration of PET and MRI brain images has met with some success [128]-[131]. Because tumors are often in locations of considerable malleability (e.g., abdomen), digital registration of independent PET and MR images can be very difficult, necessitating simultaneous imaging even for simple registration. Reliable registration at the pixel levels opens up the possibility of realistic interpretation of MR images (and changes in them) in terms of the underlying tumor physiology. Thus, the use of various $M R$ determinations $\left(T_{1}, T_{2}\right.$, contrast enhancement, ADC, metabolite concentration) as "biomarkers" indicative of changes in the tumor will be greatly facilitated. While clinical PET scans can be repeated, there are strict limits to how often, whereas MRI scans can be performed daily. Having reliable MR biomarkers that are correlated with known tumor physiological changes in the preclinical setting would be a boon to monitoring tumor response to therapeutics and optimizing treatment on a case-by-case basis. In preclinical studies, simultaneous imaging will allow assessment of the distribution of therapeutic agents by high specific activity radionuclide labeling of the agents, allowing short-term precise characterization of biodistribution and assessment of whether the mode of action is direct or indirect. Longitudinal imaging studies on the minute through week timescales are often undertaken using tumor model systems to glean information about mode(s) of action of potential therapeutics. Such repeated simultaneous PET/ MRI measurements provide temporal as well as spatial correlation of the two modalities.

Immune system-based therapies in cancer treatment are aimed at enhancing the body's own defenses. These represent a broad array of methodologies that include targeting of immunological moieties to tumor cells, activation of $\mathrm{T}$ cells, and antigen presentation [132]-[134]. Because these therapies are typically not directly acting, it is essential to monitor the time course, characteristics, and specificity of the immune response and its effect against the tumor. Both PET- and MRI-based procedures have been proposed to monitor immunotherapies [135], [136]. In terms of joint PET/MRI measurements, once again it is the qualitatively different information obtainable from the two modalities and temporal coincidence that brings real utility to the simultaneous measurement. PET agents can be used to efficiently specify the location(s) and concentration(s) of immune cell populations while modulation(s) in tumor physiology are monitored with MRI. Interrogating what is happening to the tumor while the immune response is being mounted and being able to correlate tumor changes with the duration and extent of the response will be of immense help in developing treatment protocols and in eventually personalizing treatment regimes in the clinic.

The use of dual labeled agents opens up a wealth of possibilities. For example, the rough location ( \pm millimeters) of small metastasis can be determined with PET [137], and MR can immediately provide highresolution anatomical scans of the suspect region. Although the concentration of the MR label in a small metastatic tissue volume may be too low to see in a whole body survey scan, knowledge of the location of interest gleaned from the PET image will point to the small volume(s) from which to record high-resolution MRI data using a dual labeled probe. Under these more favorable conditions, the likelihood of seeing contrast enhancement due to the label is greatly increased (especially for $\mathrm{T}_{2}$ agents). Regardless of the visibility of the label, the highresolution MR image will provide an assessment of anatomical anomalies associated with metastasis and repeated imaging will allow characterization of the time course of tumor growth and response to therapeutics from very early stages.

\section{DISCUSSION}

Instruments with the ability to simultaneously record PET and MRI measurements in both small animals and humans have been developed and are currently being refined and optimized. At the present time, PET systems based on APD technology allow existing magnets to be used and have been studied in most detail. New photondetection technologies, especially silicon or solid-state photomultiplier detectors [138], hold promise for use in PET/MRI systems with their very robust signals (which obviates the need to place preamplifiers within the magnet), fast timing, and low sensitivity to magnetic fields.

One challenge to the acceptance of PET/MRI, especially for studies in humans, is attenuation correction of the PET data. In PET/CT scanners, this correction can be derived quite directly from the CT data. Although structural MR images can in theory be segmented into major tissue types allowing known attenuation coefficients to be assigned for PET attenuation correction, it is hard to capture the inhomogeneities in density in regions such as the lung, and this could cause significant errors in the PET images. This is an ongoing and important area of investigation.

Dual-mode contrast agents for PET/MRI hold much promise but are clearly still in their infancy. Current efforts are focusing on optimizing the radio-labeling efficiency of nanoparticle-based probes, evaluating the toxicity and biodistribution of these novel probes in animal models, and initial applications, for example, in imaging of atherosclerotic plaque, using these agents with the new integrated PET/MRI instrumentation. This application requires extremely good spatial coregistration 
of the two datasets and will therefore be a good test case for simultaneous PET/MRI studies.

The future of integrated PET/MRI instruments and/or dual-mode PET/MRI contrast agents clearly depends on finding applications (whether these be in basic or clinical research, preclinical evaluation of therapeutics, or clinical diagnostics or therapeutic monitoring) that are significantly aided by simultaneous PET/MRI strategies. While some applications, such as those described here, certainly exist, the complete picture will likely not emerge for several years as the utility of combined systems becomes more widely understood.

\section{REFERENCES}

[1] M. Bottrill, L. K. Nicholas, and N. J. Long, "Lanthanides in magnetic resonance imaging," Chem. Soc. Rev., vol. 35 pp. $557-571,2006$

[2] C. Corot, P. Robert, J. M. Idee, and M. Port, "Recent advances in iron oxide nanocrystal technology for medical imaging," Adv. Drug Delivery Rev., vol. 58, pp. 1471-1504, 2006.

[3] A. M. Oros and N. J. Shah, "Hyperpolarized xenon in NMR and MRI," Phys. Med. Biol., vol. 49, pp. R105-R153, 2004.

[4] D. G. Norris, "Principles of magnetic resonance assessment of brain function," J. Magn. Res. Imag., vol. 23, pp. 794-807, 2006.

[5] D. E. Sosnovik and R. Weissleder, "Emerging concepts in molecular MRI," Curr. Opin. Biotechnol., vol. 18, pp. 4-10, 2007.

[6] S. F. Keevil, "Spatial localization in nuclear magnetic resonance spectroscopy," Phys. Med. Biol., vol. 51, pp. R579-R636, 2006.

[7] M. E. Phelps, "Positron emission tomography provides molecular imaging of biological processes," Proc. Nat. Acad. Sci., vol. 97, pp. 9226-9233, 2000.

[8] M. E. Phelps, "PET: The merging of biology and imaging into molecular imaging," J. Nucl. Med., vol. 41, pp. 661-681, 2000.

[9] D. L. G. Hill, P. G. Batchelor, M. Holden, and D. J. Hawkes, "Medical image registration," Phys. Med. Biol., vol. 46, pp. R1-R45, 2001.

[10] B. E. Hammer, N. L. Christensen, and B. G. Heil, "Use of a magnetic field to increase the spatial resolution of positron emission tomography," Med. Phys., vol. 21, pp. 1917-1920, 1994.

[11] B. E. Hammer and N. L. Christensen, "Measurement of positron range in matter in strong magnetic fields," IEEE Trans. Nucl. Sci., vol. 42, pp. 1371-1376, 1995.

[12] R. R. Raylman, B. E. Hammer, and N. L. Christensen, "Combined MRI-PET scanner: A Monte Carlo evaluation of the improvements in PET resolution due to the effects of a static homogeneous magnetic field," IEEE Trans. Nucl. Sci., vol. 43, pp. 2406-2412, 1996.

[13] A. Wirrwar, H. Vosberg, H. Herzog, H. Halling, S. Weber, and H. W. M. Gartner, " 4.5 tesla magnetic field reduces range of high-energy positrons-Potential implications for positron emission tomography," IEEE Trans. Nucl. Sci., vol. 44, pp. 184-189, 1997.

[14] S. R. Cherry, "The 2006 Henry N. Wagner lecture: Of mice and men (and positrons)-Advances in PET imaging technology," J. Nucl. Med., vol. 47, pp. 1735-1745, 2006.

[15] N. L. Christensen, B. E. Hammer, B. G. Heil, and K. Fetterly, "Positron emission tomography within a magnetic field using photomultiplier tubes and lightguides," Phys. Med. Biol., vol. 40, pp. 691-697, 1995.
[16] M. Buchanan, P. K. Marsden, C. H. Mielke, and P. B. Garlick, "A system to obtain radiotracer uptake data simultaneously with NMR spectra in a high field magnet," IEEE Trans. Nucl. Sci., vol. 43, pp. 2044-2048, 1996.

[17] Y. Shao, S. R. Cherry, K. Farahani, K. Meadors, S. Siegel, R. W. Silverman, and P. K. Marsden, "Simultaneous PET and MR imaging," Phys. Med. Biol., vol. 42, pp. 1965-1970, 1997.

[18] Y. Shao, S. R. Cherry, K. Farahani, R. Slates, R. W. Silverman, K. Meadors, A. Bowery, S. Siegel, P. K. Marsden, and P. B. Garlick, "Development of a PET detector system compatible with MRI/NMR systems," IEEE Trans. Nucl. Sci., vol. 44, pp. 1167-1171, 1997.

[19] P. B. Garlick, P. K. Marsden, A. C. Cave, H. G. Parkes, R. Slates, Y. P. Shao, R. W. Silverman, and S. R. Cherry, "PET and NMR dual acquisition (PANDA): Applications to isolated, perfused rat hearts," NMR Biomed., vol. 10, pp. 138-142, 1997.

[20] R. B. Slates, K. Farahani, S. Yiping, P. K. Marsden, J. Taylor, P. E. Summers, S. Williams, J. Beech, and S. R. Cherry, "A study of artefacts in simultaneous PET and MR imaging using a prototype MR compatible PET scanner," Phys. Med. Biol., vol. 44, pp. 2015-2027, 1999.

[21] J. E. Mackewn, D. Strul, W. A. Hallett, P. Halsted, R. A. Page, S. F. Keevil, S. C. R. Williams, S. R. Cherry, and P. K. Marsden, "Design and development of an MR-compatible PET scanner for imaging small animals," IEEE Trans. Nucl. Sci., vol. 52, pp. 1376-1380, 2005.

[22] R. R. Raylman, S. Majewski, S. K. Lemieux, S. S. Velan, B. Kross, V. Popov, M. F. Smith, A. G. Weisenberger, C. Zorn, and G. D. Marano, "Simultaneous MRI and PET imaging of a rat brain," Phys. Med. Biol., vol. 51, pp. 6371-6379, 2006.

[23] A. J. Lucas, R. C. Hawkes, R. E. Ansorge, G. B. Williams, R. E. Nutt, J. C. Clark, T. A. Fryer, and T. A. Carpenter, "Development of a combined microPET(R)-MR system," Tech. Cancer Res. Treat., vol. 5, pp. 337-341, 2006.

[24] K. M. Gilbert, W. B. Handler, T. J. Scholl, J. W. Odegaard, and B. A. Chronik, "Design of field-cycled magnetic resonance systems for small animal imaging," Phys. Med. Biol., vol. 51, pp. 2825-2841, 2006.

[25] W. B. Handler, K. M. Gilbert, H. Peng, and B. A. Chronik, "Simulation of scattering and attenuation of $511 \mathrm{keV}$ photons in a combined PET/field-cycled MRI system," Phys. Med. Biol., vol. 51, pp. 2479-2491, 2006.

[26] B. Pichler, E. Lorenz, R. Mirzoyan, W. Pimpl, F. Roder, M. Schwaiger, and S. I. Ziegler, "Performance test of a LSO-APD PET module in a 9.4 tesla magnet," presented at the IEEE Medical Imag. Conf., Albuquerque, NM, 1997.

[27] C. Catana, Y. B. Wu, M. S. Judenhofer, J. Y. Qi, B. J. Pichler, and S. R. Cherry, "Simultaneous acquisition of multislice PET and MR images: Initial results with a MR-compatible PET scanner," J. Nucl. Med., vol. 47, pp. 1968-1976, 2006.

[28] B. J. Pichler, M. S. Judenhofer, C. Catana, J. H. Walton, M. Kneilling, R. E. Nutt, S. B. Siegel, C. D. Claussen, and S. R. Cherry, "Performance test of an LSO-APD detector in a 7-T MRI scanner for simultaneous PET/MRI," J. Nucl. Med., vol. 47, pp. 639-647, 2006.

[29] C. Woody, D. Schlyer, P. Vaska, D. Tomasi, S. Solis-Najera, W. Rooney, J. F. Pratte, S. Junnarkar, S. Stoll, Z. Master, M. Purschke, S. J. Park, S. Southekal, A. Kriplani, S. Krishnamoorthy, S. Maramraju, P. O'Connor, and V. Radeka, "Preliminary studies of a simultaneous PET/MRI scanner based on the RatCAP small animal tomograph," Nucl. Instrum. Methods A, vol. 571, pp. 102-105, 2007.

[30] H.-P. W. Schlemmer, B. J. Pichler, D. W. Townsend, M. S. Judenhofer, C. Nahmias, W.-D. Heiss, and C. D. Claussen, "Combined clinical MR/PET: Feasibility of simultaneous imaging," presented at the Int. Soc. Magn. Res. Med., Berlin, Germany, 2007.

[31] N. Kawai, N. et al., "FDG-PET findings of the brain in lymphomatoid granulomatosis," Ann. Nucl. Med., vol. 20, pp. 683-687, 2006.

[32] D. B. Siepmann, A. Siegel, and P. J. Lewis, "TI-201 SPECT and F-18FDG PET for assessment of glioma recurrence versus radiation necrosis," Clin. Nucl. Med. vol. 30, pp. 199-200, 2005.

[33] K. S. Tralins et al., "Volumetric analysis of F-18-FDG PET in glioblastoma multiforme: Prognostic information and possible role in definition of target volumes in radiation dose escalation," J. Nucl. Med., vol. 43, pp. 1667-1673, 2002.

[34] A. G. Vlassenko, B. Thiessen, B. J. Beattie, M. G. Malkin, and R. G. Blasberg, "Evaluation of early response to SU101 target-based therapy in patients with recurrent supratentorial malignant gliomas using FDG PET and Gd-DTPA MRI," J. Neuro-Oncol., vol. 46, pp. 249-259, 2000.

[35] A. H. Jacobs et al., "F-18-fluoro-L-thymidine and C-11-methylmethionine as markers of increased transport and proliferation in brain tumors," J. Nucl. Med., vol. 46, pp. 1948-1958, 2005

[36] T. W. Stadnik et al., "Breast imaging. Preoperative breast cancer staging: Comparison of USPIO-enhanced MR imaging and 18F-fluorodeoxyglucose (FDC) positron emission tomography (PET) imaging for axillary lymph node staging-Initial findings," Eur. Radiol., vol. 16, pp. 2153-2160, 2006.

[37] P. M. Boiselle et al., "Imaging of mediastinal lymph nodes: CT, MR, and FDG PET," Radiographics, vol. 18, pp. 1061-1069, 1998.

[38] W. E. Palmer et al., "Quantification of inflammation in the wrist with gadolinium-enhanced MR-imaging 
and PET with 2-[F-18]-fluoro-2-deoxy-

D-glucose," Radiology, vol. 196 , pp. 647-655, 1995

[39] A. Roivainen et al., "Use of positron emission tomography with methyl-C-11-choline and 2-F-18-fluoro-2-deoxy-D-glucose in comparison with magnetic resonance imaging for the assessment of inflammatory proliferation of synovium," Arthritis Rheum. vol. 48, pp. 3077-3084, 2003.

[40] N. Sadeghi et al., "Correlation between dynamic susceptibility contrast perfusion MRI and methionine metabolism in brain gliomas: Preliminary results," J. Magn. Res. Imag., vol. 24, pp. 989-994, 2006.

[41] J. W. Koskenvuo et al., "Global myocardial blood flow and global flow reserve measurements by MRI and PET are comparable," J. Magn. Res. Imag., vol. 13, pp. 361-366, 2001

[42] J. Schwitter, "Myocardial perfusion imaging by cardiac magnetic resonance," J. Nucl. Cardiol., vol. 13, pp. 841-854, 2006.

[43] T. Higuchi et al., "Characterization of normal and infarcted rat myrocardium using a combination of small-animal PET and clinical MRI," J. Nucl. Med., vol. 48, pp. 288-294, 2007.

[44] P. Knaapen et al., "Delayed contrast enhancement and perfusable tissue index in hypertrophic cardiomyopathy," Eur. Heart J., vol. 26, pp. 387-388, 2005.

[45] H. P. Kuhl et al., "Myocardia viability in chronic ischemic heart disease-Comparison of contrast-enhanced magnetic resonance imaging with F-18-fluorodeoxyglucose positron emission tomography," J. Amer. Coll. Cardiol., vol. 41, pp. 1341-1348, 2003.

[46] C. Ceylan, U. A. van der Heide, G. H. Bol, J. J. W. Lagendijk, and A. Kotte, "Assessment of rigid multi-modality image registration consistency using the multiple sub-volume registration (MSR) method," Phys. Med. Biol., vol. 50, pp. N101-N108, 2005.

[47] D. J. Rowland, J. R. Garbow, R. Laforest, and A. Z. Snyder, "Registration of [F-18]FDG microPET and small-animal MRI," Nucl. Med. Biol., vol. 32, pp. 567-572, 2005.

[48] H. Benveniste et al., "Maternal and fetal C-11-cocaine uptake and kinetics measured in vivo by combined PET and MRI in pregnant nonhuman primates," J. Nucl. Med., vol. 46, pp. 312-320, 2005.

[49] D. G. Shen, D. F. Liu, Z. X. Cao, P. D. Acton, and R. Zhou, "Coregistration of magnetic resonance and single photon emission computed tomography images for noninvasive localization of stem cells grafted in the infarcted rat myocardium," Mol. Imag. Biol., vol. 9, pp. 24-31, 2007.

[50] R. Agger et al., "T cell homing to tumors detected by 3D-coordinated positron emission tomography and magnetic resonance imaging," J. Immun., vol. 30, pp. 29-39, 2007

[51] M. Hueber et al., "Fluorescently detectable magnetic resonance imaging agents," Bioconjugate Chem., vol. 9, pp. 42-249, 1998.

[52] V. S. Talanov et al., "Dendrimer-based nanoprobe for dual modality magnetic resonance and fluorescence imaging," Nano Lett., vol. 6, pp. 1459-1463, 2006.

[53] H. S. Yang, S. Santra, G. A. Walter, and P. H. Holloway, "Gd-III-functionalized fluorescent quantum dots as multimodal imaging probes," Adv. Mater., vol. 18, pp. $2890-2894,2006$
54] L. Josephson, M. F. Kircher, U. Mahmood, Y. Tang, and R. Weissleder, "Near-infrared fluorescent nanoparticles as combined MR/optical imaging probes," Bioconjugate Chem., vol. 13, pp. 554-560, 2002.

[55] M. F. Kircher, U. Mahmood, R. S. King, R. Weissleder, and L. A. Josephson, "A multimodal nanoparticle for preoperative magnetic resonance imaging and intraoperative optical brain tumor delineation," Cancer Res., vol. 63 , pp. 8122-8125, 2003.

[56] Z. Medarova, W. Pham, Y. Kim, G. P. Dai, and A. Moore, "In vivo imaging of tumor response to therapy using a dual-modality imaging strategy," Int. J. Cancer, vol. 118, pp. 2796-2802, 2006

[57] E. A. Schellenberger, D. Sosnovik, R. Weissleder, and L. Josephson, "Magneto/ optical annexin V, a multimodal protein," Bioconjugate Chem., vol. 15, pp. 1062-1067, 2004

[58] D. E. Sosnovik et al., "Magnetic resonance imaging of cardiomyocyte apoptosis with a novel magneto-optical nanoparticle," Magn. Res. Med., vol. 54, pp. 718-724, 2005.

[59] Y. M. Huh et al., "In vivo magnetic resonance detection of cancer by using multifunctional magnetic nanocrystals," J. Amer. Chem. Soc., vol. 127, pp. 12387-12391, 2005.

[60] S. Wang, B. R. Jarrett, S. M. Kauzlarich, and A. Y. Louie, "Core/shell quantum dots with high relaxivity and photoluminescence for multimodality imaging," J. Amer. Chem. Soc., vol. 129, pp. 3848-3856, 2007.

[61] H. C. Lu et al., "Synthesis and characterization of multi-functional nanoparticles possessing magnetic, up-conversion fluorescence and bio-affinity properties," J. Mater. Chem., vol. 14, pp. 1336-1341, 2004.

[62] T. J. Yoon et al., "Multifunctional nanoparticles possessing a 'magnetic motor effect' for drug or gene delivery," Angewandte Chem., vol. 44, pp. 1068-1071, 2005, Int. Ed.

[63] H. W. Gu, R. K. Zheng, X. X. Zhang, and B. Xu, "Facile one-pot synthesis of bifunctional heterodimers of nanoparticles: A conjugate of quantum dot and magnetic nanoparticles," J. Amer. Chem. Soc., vol. 126 , pp. 5664-5665, 2004

[64] J. S. Choi et al., "Biocompatible heterostructured nanoparticles for multimodal biological detection," J. Amer. Chem. Soc., vol. 128, pp. 15 982-15 983, 2006.

[65] S. Santra, S. et al., "Synthesis and characterization of fluorescent, radio-opaque, and paramagnetic silica nanoparticles for multimodal bioimaging applications," Adv. Mater., vol. 17, pp. 2165-2169, 2005

[66] V. Humblet, P. Misra, and J. Frangioni, "An HPLC/mass spectrometry platform for the development of multimodality contrast agents and targeted therapeutics: Prostate-specific membrane antigen small molecule derivatives," Contrast Media Mol. Imag., vol. 1, pp. 196-211, 2006.

[67] C. Li et al., "Dual optical and nuclear imaging in human melanoma xenografts using a single targeted imaging probe," Nucl. Med Biol., vol. 33, pp. 349-358, 2006.

[68] B. Gustafsson, S. Youens, and A. Y. Louie, "Development of contrast agents targeted to macrophage scavenger receptors for MRI of vascular inflammation," Bioconjugate Chem., vol. 17, pp. 538-547, 2006

[69] B. Jarrett, M. Frendo, J. Vogan, and A. Louie, "Size controlled synthesis of dextran sulfate coated iron oxide nanoparticles for magnetic resonance imaging," Nanotechnology, vol. 18, p. 035603, 2007.

[70] R. Silverstein and M. Febbraio, "CD36 and atherosclerosis," Current Opinion Lipidol., vol. 11, pp. 482-491, 2000.

[71] B. Trigatti, A. Rigotti, and A. Braun, "Cellular and physiological roles of SR-BI, a lipoprotein receptor which mediates selective lipid uptake," Biochim. Biophys. Acta, vol. 1-3, pp. 276-286, 2000.

[72] M. Sakai, S. Kobori, A. Miyazaki, and S. Horiuchi, "Macrophage proliferation in atherosclerosis," Current Opinion Lipidol., vol. 11, pp. 503-509, 2000

[73] P. Gough et al., "Analysis of macrophage scavenger receptor (SR-A) expression in human aortic atherosclerotic lesions," Arterioscler. Thromb. Vasc. Biol., vol. 19, pp. 461-471, 1999.

[74] M. Linton and S. Fazio, "Class A scavenger receptors, macrophages and atherosclerosis," Current Opinion Lipidol. vol. 12, pp. 489-495, 2001.

[75] M. deWinther, K. vanDijk, L. Havekes, and M. Hofker, "Macrophage scavenger receptor class A," Arterioscler. Thromb. Vasc. Biol., vol. 20, pp. 290-297, 1999.

[76] P. Caravan, J. Ellison, T. McMurry, and R. Lauffer, "Gadolinium (III) chelates as MRI contrast agents: Structure, dynamics, and applications," Chem. Rev., vol. 99, pp. 2293-2352, 1999.

[77] P. Blower, J. Lewis, and J. Zweit, “Copper radionuclides and radiopharmaceuticals in nuclear medicine," Nucl. Med. Biol., vol. 23, pp. 957-980, 1996.

[78] M. F. Tweedle, "Physicochemical properties of gadoteridol and other magnetic resonance contrast agents," Invest. Radiol. vol. 27, pp. S2-S6, 1992.

[79] L. Thunus and R. Lejeune, "Overview of transition metal and lanthanide complexes as diagnostic tools," Coord. Chem. Rev. vol. 184, pp. 125-155, 1999.

[80] T. Nagae, A. Louie, S. Aizawa, S. Ishimaru, and S. Wilson, "Selective targeting and photodynamic destruction of intimal hyperplasia by scavenger-receptor mediated protein-chlorin e6 conjugates," J. Cardio. Surg., vol. 39, pp. 709-715, 1998.

[81] P. Butler and B. Hartley, "Maleylation of proteins," Meth. Enzymol., vol. 25, pp. 191-199, 1972.

[82] S. Mirzadeh, M. W. Brechbiel, R. W. Atcher, and O. A. Gansow, "Radiometal labeling of immunoproteins: Covalent linkage of 2-(4-isothiocyanatobenzyl)diethylenetriaminepentaacetic acid ligands to immunoglobulin," Bioconjugate Chem., vol. 1, pp. 59-65, 1990.

[83] J. Lewis, R. Laforest, M. Lewis, and C. Anderson, "Comparative dosimetry of copper-64 and yttirum-90-labeled comatostatin analogs in a tumor-bearing rat model," Cancer Biotherapy Radiopharm. vol. 15, pp. 593-604, 2000

[84] U. Kragh-Hansen, "Structure and ligand binding properties of human serum albumin," Dan. Med. Bull., vol. 37, pp. 57-84, 1990.

[85] I. Cicha et al., "Connective tissue growth factor is overexpressed in comlicated atherosclerotic plaques and induced mononuclear cell chemotaxis in vitro," Arterioscler. Thromb. Vasc. Biol., vol. 25 , pp. 1008-1013, 2005.

[86] C. Chapon et al., "High field magnetic resonance imaging evaluation of 
superparamagnetic iron oxide nanoparticles in a permanent rat myocardial infarction," Inv. Radiol., vol. 38, pp. 141-146, 2003.

[87] A. W. Toga and P. M. Thompson, "The role of image registration in brain mapping," Image Vision Comput., vol. 19, pp. 3-24, 2001

[88] I. K. Lyoo and P. F. Renshaw, "Magnetic resonance spectroscopy: Current and future applications in psychiatric research," Biol. Psych., vol. 51, pp. 195-207, 2002.

[89] W. Wolf, C. A. Presant, and V. Waluch, "F-19-MRS studies of fluorinated drugs in humans," Adv. Drug Delivery Rev., vol. 41, pp. 55-74, 2000.

[90] H. X. Lei, F. Morgenthaler, T. W. Yue, and R. Gruetter, "Direct validation of in vivo localized C-13 MRS measurements of brain glycogen," Magn. Res. Med., vol. 57, pp. 243-248, 2007.

[91] D. K. Deelchand, K. Ugurbil, and P. G. Henry, "Investigating brain metabolism at high fields using localized C-13 NMR spectroscopy without H-1 decoupling," Magn. Res. Med., vol. 55, pp. 279-286, 2006.

[92] R. A. de Graaf, G. F. Mason, A. B. Patel, D. L. Rothman, and K. L. Behar, "Regional glucose metabolism and glutamatergic neurotransmission in rat brain in vivo," Proc. Nat. Acad. Sci. USA, vol. 101, pp. 12700-12705, 2004.

[93] G. Brix, M. E. Bellemann, U. Haberkorn, L. Gerlach, and W. J. Lorenz, "Assessment of the biodistribution and metabolism of 5-fluorouracil as monitored by 18F PET and 19F MRI: A comparative animal study," Nucl. Med. Biol, vol. 23, pp. 897-906, 1996.

[94] A. Srinivasan, S. S. Gambhir, J. R. Barrio, L. Wu, M. Namavari, N. Satyamurthy, S. Sharfstein, S. Cherry, A. L. Green, A. Berk, M. E. Phelps, and H. R. Herschman, "Imaging reporter gene expression in living animals with positron emission tomography clinical application to gene therapy," Blood, vol. 88, pp. 1717-1717, 1996.

[95] S. S. Gambhir, H. R. Herschman, S. R. Cherry, J. R. Barrio, N. Satyamurthy, T. Toyokuni, M. E. Phelps, S. M. Larson, J. Balatoni, R. Finn, M. Sadelain, J. Tjuvajev, and R. Blasberg, "Imaging transgene expression with radionuclide imaging technologies," Neoplasia, vol. 2, pp. 118-138, 2000.

[96] X. Sun, A. J. Annala, S. S. Yaghoubi, J. R. Barrio, K. N. Nguyen, T. Toyokuni, N. Satyamurthy, M. Namavari, M. E. Phelps, H. R. Herschman, and S. S. Gambhir, "Quantitative imaging of gene induction in living animals," Gene Therapy, vol. 8, pp. 1572-1579, 2001.

[97] V. Sharma and D. Piwnica-Worms, "Molecular imaging of gene expression and protein function in vivo with PET and SPECT," J. Magn. Res. Imag., vol. 16, pp. 336-351, 2002.

[98] A. H. Kesarwala, J. L. Prior, J. W. Sun, S. E. Harpstrite, V. Sharma, and D. Piwnica-Worms, "Second-generation triple reporter for bioluminescence, micro-positron emission tomography, and fluorescence imaging," Mol. Imag., vol. 5, pp. 465-474, 2006

[99] A. W. Toga and J. C. Mazziotta, Brain Mapping: The Methods, 2nd ed. San Diego, CA: Academic, 2002.

[100] A. W. Toga and J. C. Mazziotta, Brain Mapping-The Systems. San Diego, CA: Academic, 2000.
[101] L. Ostergaard, D. F. Smith, P. Vestergaard-Poulsen, S. B. Hansen, A. D. Gee, A. Gjedde, and C. Gyldensted, "Absolute cerebral blood flow and blood volume measured by magnetic resonance imaging bolus tracking: Comparison with positron emission tomography values," J. Cerebral Blood Flow Metab., vol. 18, pp. 425-432, 1998.

[102] P. Girardi and F. Dassie, "Compromise of the attention of the working memory in subjects with early episody: A study of fMRI and PET with the paradigm of the tower of London (TOL)," Schizophrenia Res., vol. 86, pp. S27-S28, 2006.

[103] K. F. Mann, M. Smolka, and A. Heinz, "Induced brain activity in alcoholics: A combined PET and fMRI study," Alcoholism-Clin. Exper. Res., vol. 30 pp. 100A-100A, 2006.

[104] P. Pietrini, E. Ricciardi, M. Guazzelli, and M. Furey, "Neuronal correlates of cholinergic enhancement on cognitive function: in vivo PET and fMRI studies in humans," J. Psychophysiol., vol. 20 pp. 214-214, 2006.

[105] S. G. Danko, N. P. Bechtereva, N. V. Shemyakina, S. V. Medvedev, and S. V. Pahomov, "Combining PET/fMRI and EEG methods in studies of certain mental states," Int. J. Psychophysiol., vol. 61, pp. 324-324, 2006.

[106] H. Ito, M. Ibaraki, I. Kanno, H. Fukuda, and S. Miura, "Changes in cerebral blood flow and cerebral oxygen metabolism during neural activation measured by positron emission tomography: Comparison with blood oxygenation level-dependent contrast measured by functional magnetic resonance imaging," J. Cerebral Blood Flow Metab., vol. 25, pp. 371-377, 2005

[107] A. Malizia, "Imaging GABA brain function in man with PET and fMRI," J. Psychopharmacol., vol. 19, pp. A6-A6, 2005.

[108] A. B. Newberg, J. J. Wang, H. Y. Rao, R. L. Swanson, N. Wintering, J. S. Karp, A. Alavi, J. H. Greenberg, and J. A. Detre, "Concurrent CBF and CMRGlc changes during human brain activation by combined fMRI-PET scanning," Neuroimage, vol. 28, pp. 500-506, 2005.

[109] A. M. Owen, M. R. Coleman, D. K. Menon, I. S. Johnsrude, J. M. Rodd, M. H. Davis, K. Taylor, and J. D. Pickard, "Residual auditory function in persistent vegetative state: A combined PET and fMRI study," Neuropsychol. Rehab., vol. 15, pp. 290-306, 2005.

[110] N. Sadato, Y. Yonekura, H. Yamada, S. Nakamura, A. Waki, and Y. Ishii, "Activation patterns of covert word generation detected by fMRI: Comparison with 3D PET," J. Comput. Assist. Tomogr., vol. 22, pp. 945-952, 1998.

[111] J. T. Coull and A. C. Nobre, "Where and when to pay attention: The neural systems for directing attention to spatial locations and to time intervals as revealed by both PET and fMRI," J. Neurosci., vol. 18, pp. 7426-7435, 1998.

[112] J. G. Ojemann, R. L. Buckner, E. Akbudak, A. Z. Snyder, J. M. Ollinger, R. C. McKinstry, B. R. Rosen, S. E. Petersen, M. E. Raichle, and T. E. Conturo, "Functional MRI studies of word-stem completion: Reliability across laboratories and comparison to blood flow imaging with PET," Human Brain Mapping, vol. 6, pp. 203-215, 1998.

[113] J. R. Votaw, T. L. Faber, C. A. Popp, T. R. Henry, J. D. Trudeau, J. L. Woodard,
H. Mao, R. M. Woods, A. W. Song, and J. M. Hoffman, "Naming task shows congruent activations and deactivations: A combined PET and fMRI study," J. Nucl. Med., vol. 39, pp. 110P-110P, 1998.

[114] J. H. Xiong, S. Rao, J. H. Gao, M. Woldorff, and P. T. Fox, "Evaluation of hemispheric dominance for language using functional MRI: A comparison with positron emission tomography," Human Brain Mapping, vol. 6, pp. 42-58, 1998.

[115] G. Rees, A. Howseman, O. Josephs, C. D. Frith, K. J. Friston, R. S. J. Frackowiak, and R. Turner, "Characterizing the relationship between BOLD contrast and regional cerebral blood flow measurements by varying the stimulus presentation rate," Neuroimage, vol. 6 , pp. 270-278, 1997.

[116] K. J. Worsley, J. B. Poline, K. J. Friston, and A. C. Evans, "Characterizing the response of PET and fMRI data using multivariate linear models," Neuroimage, vol. 6, pp. 305-319, 1997.

[117] T. V. Nguyen, A.-L. Brownell, Y.-C. I. Chen, E. Livni, J. T. Coyle, B. R. Rosen, F. Cavagna, and B. G. Jenkins, "Detection of the effects of dopamine receptor supersensitivity using pharmacological MRI and correlations with PET," Synapse, vol. 36, pp. 57-65, 2000.

[118] Y. C. Chen, W. R. Galpern, A. L. Brownell, R. T. Matthews, M. Bogdanov, O. Isacson, J. R. Keltner, M. F. Beal, B. R. Rosen, and B. G. Jenkins, "Detection of dopaminergic neurotransmitter activity using pharmacologic MRI: Correlation with PET, microdialysis, and behavioral data," Magn. Res. Med., vol. 38, pp. 389-398, 1997.

[119] B. J. Nieman, A. M. Flenniken, S. L. Adamson, R. M. Henkelman, and J. G. Sled, "Anatomical phenotyping in the brain and skull of a mutant mouse by magnetic resonance imaging and computed tomography," Physiol. Genomics, vol. 24, pp. 154-162, 2006.

[120] M. K. Simmons, R. Manjeshwar, E. D. Agdeppa, R. M. Mattheyses, T. R. Kiehl, and M. C. Montalto, "A computational positron emission tomography simulation model for imaging beta-amyloid in mice," Mol. Imag. Biol., vol. 7, pp. 69-77, 2005.

[121] J. L. Goggi, H. D. Lewis, J. Mok, T. Harrison, M. S. Shearman, J. R. Atack, and J. D. Best, "A comparative assessment of gamma-secretase activity in transgenic and non-transgenic rodent brain," J. Neurosci. Meth., vol. 157, pp. 246-252, 2006.

[122] M. J. Welch, J. S. Lewis, J. Kim, T. L. Sharp, C. S. Dence, R. J. Gropler, and P. Herrero, "Assessment of myocardial metabolism in diabetic rats using small-animal PET: A feasibility study," J. Nucl. Med., vol. 47, pp. 689-697, 2006.

[123] J. Horacek, F. Spaniel, M. Kopecek, T. Novak, M. Brunovsky, C. Dockery, and C. Hoschl, "The influence of polymorphism for regulator of G-protein signaling 4 (RGS4) gene on regional brain metabolism (18FDG PET) and phenotypes in schizophrenia," Schizophrenia Res., vol. 81, pp. 220-220, 2006.

[124] R. Lim, F. H. Fahey, L. A. Drubach, L. P. Connolly, and S. T. Treves, "Early experience with fluorine-18 sodium fluoride bone PET in young patients with back pain," J. Ped. Ortho., vol. 27, pp. 277-282, 2007. 
[125] M. L. Frost, G. J. R. Cook, G. M. Blake, P. K. Marsden, and I. Fogelman, "The relationship between regional bone turnover measured using F-18-fluoride positron emission tomography and changes in BMD is equivalent to that seen for biochemical markers of bone turnover," J. Clin. Dens., vol. 10, pp. 46-54, 2007.

[126] O. K. Oz, G. Hirasawa, J. Lawson, L. Nanu, A. Constantinescu, P. P. Antich, R. P. Mason, E. Tsyganov, R. W. Parkey, J. E. Zerwekh, and E. R. Simpson, "Bone phenotype of the aromatase deficient mouse," J. Steroid Biochem. Mol. Biol., vol. 79, pp. 49-59, 2001.

[127] F. W. Wehrli, P. K. Saha, B. R. Gomberg, and H. K. Song, "Noninvasive assessment of bone architecture by magnetic resonance micro-imaging-based virtual bone biopsy," Proc. IEEE, vol. 91, pp. 1520-1542, 2003.

[128] M. C. Preul, R. Leblanc, Z. Caramanos, R. Kasrai, S. Narayanan, and D. L. Arnold, "Magnetic resonance spectroscopy guided brain tumor resection: Differentiation between recurrent glioma and radiation change in two diagnostically difficult cases," Can. J. Neurol. Sci., vol. 25, pp. 13-22, 1998.

[129] R. P. Woods, S. T. Grafton, C. J. Holmes, S. R. Cherry, and J. C. Mazziotta,
"Automated image registration-I. General methods and intrasubject, intramodality validation," J. Comput. Assist. Tomogr., vol. 22, pp. 139-152, 1998.

[130] R. P. Woods, S. T. Grafton, J. D. Watson, N. L. Sicotte, and J. C. Mazziotta, "Automated image registration: II. Intersubject validation of linear and nonlinear models," J. Comput. Assist. Tomogr., vol. 22, pp. 153-165, 1998.

[131] N. Hayakawa, K. Uemura et al., "A PET-MRI registration technique for PET studies of the rat brain," Nucl. Med. Biol., vol. 17, pp. 121-125, 2000.

[132] P. K. Srivastava, "Tumour immunology," Current Opinion Immunol., vol. 18, pp. 199-200, 2006.

[133] C. Baecher-Allan and D. E. Anderson, "Immune regulation in tumor-bearing hosts," Current Opinion Immunol., vol. 18, pp. 214-219, 2006.

[134] I. H. Pastan and R. J. Kreitman, "Immunotoxins in cancer therapy," Current Opinion Inv. Drugs, vol. 3, pp. 1089-1091, 2002.

[135] D.-E. Hu, M. I. Kettunen, and K. M. Brindle, "Monitoring T-lymphocyte trafficking in tumors undergoing immune rejection,"
Magn. Res. Med., vol. 54, pp. 1473-1479, 2005.

[136] C. J. Shu, S. Guo, Y. J. Kim, S. M. Shelly, A. Nijagal, P. Ray, S. S. Gambhir, C. G. Radu, and O. N. Witte, "Visualization of a primary anti-tumor immune response by positron emission tomography," PNAS, vol. 102, pp. 17412-17417, 2005.

[137] G. W. Philpott, S. W. Schwarz,

C. J. Anderson, F. Dehdashti, J. M. Connett, K. R. Zinn, C. F. Meares, P. D. Cutler, M. J. Welch, and B. A. Siegel,

"RadioimmunoPET: Detection of

colorectal carcinoma with positron-emitting copper-64-labeled monoclonal antibody," J. Nucl. Med., vol. 36, pp. 1818-1824, 1995

[138] D. P. McElroy, V. Saveliev, A. Reznik, and J. A. Rowlands, "Evaluation of silicon photomultipliers: A promising new detector for MR compatible PET," Nucl. Instrum. Methods A, vol. 571, pp. 106-109, 2007.

[139] S. R. Cherry, "Multimodality imaging systems: Twice the power or double the trouble?" Ann. Rev. Biomed. Eng., vol. 8, pp. 35-62, 2006

\section{ABOUT THE AUTHORS}

Simon R. Cherry (Fellow, IEEE) received the B.Sc. (Hons.) degree in physics with astronomy, University College London, U.K., in 1986 and the Ph.D. degree in medical physics from the Institute of Cancer Research, University of London, U.K., in 1989.

$\mathrm{He}$ is currently a Professor of biomedical engineering and Director of the Center for Molecular and Genomic Imaging, University of California, Davis. His research interests focus on developing new in vivo imaging technologies with an emphasis on positron emission tomography (PET) and fluorescence imaging, and the integration of PET with computed tomography and magnetic resonance imaging.

Dr. Cherry is a founding member of the Society for Molecular Imaging and a member of the Academy of Molecular Imaging. He was invited to present the 2006 Henry Wagner Distinguished Lectureship at the 2006 Society of Nuclear Medicine Conference. He received the IEEE Nuclear and Plasma Sciences Society Early Achievement Award in 1998.

Angelique Y. Louie received the B.S. degree from the University of California, Davis, and the M.S. degree from the University of California, Los Angeles, both in electrical engineering. He received the Ph.D. degree in biological sciences from the University of California, Irvine.

She held a Postdoctoral Fellowship at the California Institute of Technology, Pasadena. She is currently an Associate Professor in the Department of Biomedical Engineering, University of

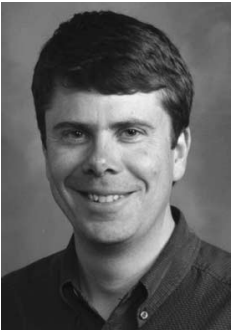

E. Jacobs received the B.S. degree in chemistry and philosophy from Tulane University, New Orleans, LA, and the Ph.D. degree in physical chemistry from Stanford University, Stanford, CA.

He held a Postdoctoral Fellowship at the University of Illinois, Urbana-Champaign. He is a member of the Beckman Institute, California Institute of Technology, Pasadena. His research focuses on the development and application of high-resolution MRI in animal models of disease and brain development. Recent work from his lab describes the use of $\mathrm{Mn}^{2+}$ to assess changes in neuronal transport in neurodegenerative disease and applications of MRI to examine morphogenic movements in the early amphibian embryo.

Dr. Jacobs is a member of the Society for Developmental Biology, American Chemical Society, Society for Neurosciences, Biophysical Society, International Society for Magnetic Resonance in Medicine, Ampere Society, and American Association for the Advancement of Science. 